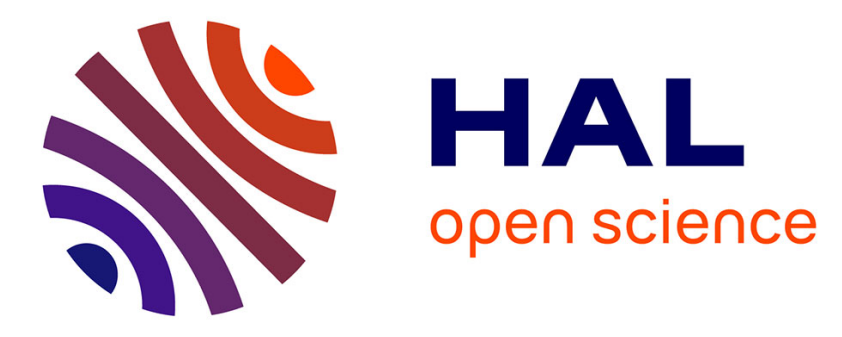

\title{
Investigation of flow structures involved in sound generation by two- and three-dimensional cavity flows
}

Philippe Druault, Xavier Gloerfelt, Thomas Mervant

\section{To cite this version:}

Philippe Druault, Xavier Gloerfelt, Thomas Mervant. Investigation of flow structures involved in sound generation by two- and three-dimensional cavity flows. Computers and Fluids, 2011, 48 (1), pp.54-67. 10.1016/j.compfluid.2011.03.014 . hal-01069687

\section{HAL Id: hal-01069687 \\ https://hal.science/hal-01069687}

Submitted on 29 Sep 2014

HAL is a multi-disciplinary open access archive for the deposit and dissemination of scientific research documents, whether they are published or not. The documents may come from teaching and research institutions in France or abroad, or from public or private research centers.
L'archive ouverte pluridisciplinaire HAL, est destinée au dépôt et à la diffusion de documents scientifiques de niveau recherche, publiés ou non, émanant des établissements d'enseignement et de recherche français ou étrangers, des laboratoires publics ou privés. 


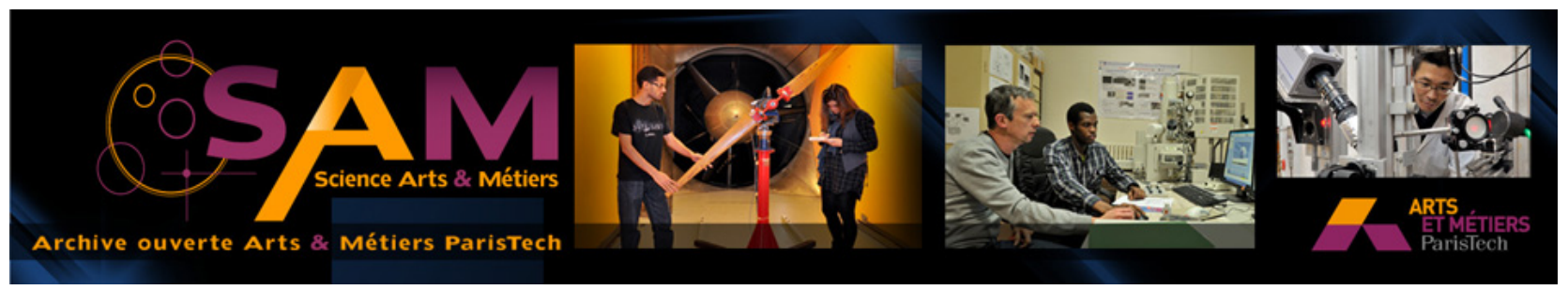

Science Arts \& Métiers (SAM)

is an open access repository that collects the work of Arts et Métiers ParisTech researchers and makes it freely available over the web where possible.

This is an author-deposited version published in: http://sam.ensam.eu

Handle ID: .http://hdl.handle.net/10985/8645

\section{To cite this version :}

Philippe DRUAULT, Xavier GLOERFELT, Thomas MERVANT - Investigation of flow structures involved in sound generation by two- and three-dimensional cavity flows - Computers \& Fluids Vol. 48, n¹, p.54-67 - 2011 


\title{
Investigation of flow structures involved in sound generation by two- and three-dimensional cavity flows
}

\author{
Philippe Druault ${ }^{\mathrm{a}}$, Xavier Gloerfelt ${ }^{\mathrm{b}}$, Thomas Mervant ${ }^{\mathrm{b}}$ \\ ${ }^{a}$ University Pierre et Marie Curie-Paris 6, Institut Jean Le Rond d'Alembert UMR CNRS 7190, case 162, 75252 Paris \\ cedex 5-France \\ ${ }^{b}$ Arts et Métiers ParisTech, DynFluid Laboratory, 75013 Paris-France
}

\begin{abstract}
Proper Orthogonal Decomposition and Stochastic Estimation are combined to shed some light on the link between organized flow structures and noise generation by turbulent flows. Proper Orthogonal Decomposition (POD) is firstly used to extract selected flow events. Based on the knowledge of these structures, the Quadratic Stochastic Estimation of the acoustic pressure field is secondly performed. Both procedures are successively applied to two- and three-dimensional numerical databases of a flow over a cavity. It is demonstrated that POD can extract selected aerodynamic events which can be associated with selected frequencies in the acoustic spectra. Reconstructed acoustic fields also indicate the aerodynamic events which are responsible of the main energy of the noise emission. Such mathematical tools offer new perspectives in analysing flow structures involved in sound generation by turbulent flows and in the experimental design of a flow control strategy.
\end{abstract}

Keywords: turbulent flow, Proper Orthogonal Decomposition, Stochastic Estimation,

\section{Introduction}

Nowadays, in the transport industry, the reduction of noise and the enhancement of sound comfort have become a commercial and economic stake of foreground. One of the major issue in such industrial applications concerns the question of noise generated by turbulent flows. The great challenge consists in relating the acoustic pressure in the farfield to its corresponding signature in the aerodynamic nearfield.

Lighthill[1] was the first to propose a reformulation of the governing equations of fluid dynamics into a wave propagation equation. This reformulation called Lighthill's analogy[1] allows 
a description of the sound field generated by turbulent flows. For this purpose, the nearfield solution is calculated with a first simulation of the unsteady fluid flow to compute the source term used for the evaluation of the wave equation. The definition of this source term has to be properly specified to enhance the reliability of such hybrid approaches. Even if many researchers proposed improved formulations for this source term, some controversies related to the physical meaning of an aeroacoustic source have been noted in the literature [2, 3, 4, 5, 6, 7]. It thus appears that such hybrid approaches can only be justified under certain idealized flow conditions. Moreover, the noise source modelling is directly related to the understanding of the turbulent flow which still remains an open issue. Such difficulty is also present when dealing with Direct Noise Computations (DNC) where both the turbulent flow and the radiated acoustic field are computed in the same run. In such numerical simulations, isolating the flow structures which are responsible of the noise emission remains a great challenge, since it is still unclear how individual flow structures contribute to the noise generation process. In turbulent flows, many flow structures of different scales coexist and interact, and they are embedded within a randomly distributed field. Since an universal and unique definition of a flow structure does not exist, the flow structure extraction still remains a difficult task. Consequently, there is still a need for the development of post-processing tools aiming at accessing the aerodynamic events which govern noise production in turbulent flows.

Previous experimental studies have been devoted to the characterization of the correlations which exist between the radiated acoustic and aerodynamic fields $[8,9,10,11]$. These works based on the causality approach have improved our knowledge of the noise source mechanisms in turbulent flows[10, 11]. They also underlined the difficulty in identifying and isolating the flow structures which are involved in the noise generation by turbulent flows. Recent numerical aeroacoustic analyses $[12,13]$ confirmed the previous experimental investigations by correlating the aerodynamic and acoustic fields.

Recently, based on experimental measurements in a flow over a cylinder, Henning et al. [14] proposed to apply the Proper Orthogonal Decomposition (POD) technique in order to identify the POD eigenfunctions which are mainly involved in the sound generation process. In a similar way, Druault et al.[15] proposed to combine POD and Quadratic Stochastic Estimation (QSE) methods to determine the aerodynamic events which contribute to the far field noise, in a forced two-dimensional compressible plane mixing layer flow. They proposed to separate the flow 
structures into three parts: large scale structures, small scale ones and background fluctuations. Each of this flow contribution was used as conditional event to QSE estimate the corresponding acoustic pressure spectrum. A direct investigation of the acoustic field as a function of different flow event was then possible. Following these premilinary developments, new applications of these mathematical post-processing tools (POD and QSE) are investigated in this paper. We focus on a three dimensional DNC solver allowing the direct evaluation of the sound occurring in the well-documented cavity flow. Based on DNC simulation databases, POD and QSE are implemented to analyze the relevant flow structures which are best correlated to the acoustic pressure field in a statistical sense. The objective of the application of the QSE procedure is not to access the individual aeroacoustic sources generated by the cavity turbulent flow. The purpose of the work rather consists in linking the statistical organized structures defined thanks to the POD partitioning, to the frequency peaks of the acoustic spectrum.

Cavity flow which occurs in many practical automotive and aerospace applications, has been studied in numerous investigations in the past (see for instance review articles $[16,17,18,19$, 20]). Recall briefly that the noise spectrum of cavity flow comprises broadband components, due to the turbulence in the shear layer and tonal components due to the periodical vortex shedding from the cavity leading edge which gives rise to intense self-sustained oscillations. These oscillations arise from a feedback loop consisting in the following chain of events. The growth and convection of instability waves in the shear layer induce large-amplitude pressure disturbances as the large scale coherent structures impinge the downstream corner of the cavity. The upstream influence of the generated pressure fluctuations provides further excitation of the instabilities in the shear layer, especially in its most receptive region near the upstream edge. A stable phase criterion is then installed between the downstream and the upstream edges of the cavity. Existing 2-D and 3-D DNC databases for low Reynolds-number flow over rectangular cavities [21, 22] are used to link selected flow structure events and acoustic pressure fields.

The paper is organized as follows. In Section 2, the post-processing tools (POD and QSE procedures) including the coupling of both procedures are briefly recalled. In section 3, the Navier Stokes solver and the flow configuration are presented. Section 4 shows a coupled POD-QSE aeroacoustic analysis of a 2-D cavity flow database. Finally, section 5 deals with an application to a 3-D cavity flow database. 


\section{Mathematical tools}

Before describing the Proper Orthogonal Decomposition and Quadratic Stochastic Estimation procedures, the following notations are introduced: $u_{i}$ and $p$ correspond to the $i^{\text {th }}$ fluctuating velocity component and to the fluctuating pressure field respectively. These fluctuating variables are directly deduced from the classical Reynolds decomposition.

\subsection{Proper Orthogonal Decomposition}

The Proper Orthogonal Decomposition is a well-known technique for determining an optimal basis for the reconstruction of a data set. This technique has been used in the past in numerous fields of application. For instance, in fluid mechanics, Lumley [23] suggested to use POD for extracting coherent structures from turbulent flows. He then proposed to define a coherent structure as the one having the largest mean square projection on the velocity field. They are consequently the solutions of the following Fredholm integral eigenvalue problem [24]:

$$
\sum_{j=1}^{N_{c}} \int_{\mathcal{D}} R_{i j}\left(\mathbf{x}, \mathbf{x}^{\prime}\right) \phi_{j}^{(n)}\left(\mathbf{x}^{\prime}\right) d \mathbf{x}^{\prime}=\lambda^{(n)} \phi_{i}^{(n)}(\mathbf{x}),
$$

where $N_{c}$ is the total number of velocity components taken into account, $\mathbf{x}$ denotes the spatial coordinates and $\mathcal{D}$ is the spatial domain under consideration. $R_{i j}\left(\mathbf{x}, \mathbf{x}^{\prime}\right)$ corresponds the timeaveraged two-point spatial correlation tensor. In this flow decomposition, $\phi_{j}^{(n)}$ is the $n^{\text {th }}$ POD eigenmode associated with the $j^{\text {th }}$ velocity component and $\lambda^{(n)}$ is the corresponding POD eigenvalue. Sirovich [25] proposed an equivalent approach called snapshot POD for the cases where the kernel $R_{i j}\left(\mathbf{x}, \mathbf{x}^{\prime}\right)$ corresponds to a high-dimensional tensor. In this approach, the temporal POD modes $a^{(n)}$ are computed from the spatial-averaged fluctuating velocity correlation tensor, and each instantaneous velocity component is expressed as follows:

$$
u_{i}(\mathbf{x}, t)=\sum_{n=1}^{N_{\text {mod }}} a^{(n)}(t) \phi_{i}^{(n)}(\mathbf{x}),
$$

where $t$ is the time variable, $\phi_{j}^{(n)}$ corresponds to the POD coefficient projected onto the $a^{(n)}$ POD mode and $N_{\text {mod }}$ is the total number of POD modes. These POD modes are orthonormal, i.e. $\left\langle\phi_{j}^{(n)} \phi_{j}^{(m)}\right\rangle=\lambda^{(n)} \delta_{n m}$, with $\delta_{n m}$ the Kronecker symbol and \langle\rangle indicates a spatial average. The coefficients are uncorrelated, i.e. $\overline{a^{(n)}(t) a^{(m)}(t)}=\delta_{n m}$, the overbar indicating a time average. 


\subsection{Stochastic Estimation}

From a global point of view, the stochatic estimation is the approximation (or estimation) of a random variable in terms of some other random variables which are known. Numerous applications of such procedures have been performed in many disciplines. For instance, in fluid mechanics, the stochatic estimation has been introduced by Adrian [26] in order to provide a conditional estimate of the large-scale structures present in turbulent flows. In this context, the estimation uses a specified conditional event about the flow at one or more locations together with its statistical properties to estimate the information at surrounding locations [27, 28, 29, 30]. A lot of previous applications have shown the efficiency of the linear version (Linear Stochastic Estimation, LSE) to detect and extract the large scale coherent structures of turbulent flows [29, 31]. However, the linear approximation seems unlikely to be able to describe a strongly non-linear phenomenon, especially when dealing with two different flow variables (velocity and pressure for instance) $[15,27,30,32]$. In the latter case, the extension to the second order of the stochastic estimation is generally retained. Note that we have previously observed that for a highly coherent 2-D cavity flow such as the one studied in the section 4, the LSE and QSE estimations of the acoustic pressure field from the knowledge of the aerodynamic flow can provide quite similar results [33]. We only observed some differences on the acoustic pressure levels which were slightly attenuated when using LSE procedure. Based on this work and on the literature results, the Quadratic Stochastic Estimation (QSE) is subsequently used in the present work.

Mathematically, based on the knowledge of instantaneous velocity field $\mathbf{u}$ at selected flow sensors, referenced with ref subscripts, the QSE estimation of the acoustic pressure $\hat{p}$ at location $\boldsymbol{x}^{\prime}$ is given by:

$$
\hat{p}\left(\boldsymbol{x}^{\prime}\right)=A_{j}\left(\boldsymbol{x}^{\prime}, \boldsymbol{x}_{\boldsymbol{r e f}}\right) u_{j}\left(\boldsymbol{x}_{\boldsymbol{r e f}}\right)+B_{j k}\left(\boldsymbol{x}^{\prime}, \boldsymbol{x}_{1 \text { ref }}, \boldsymbol{x}_{\mathbf{2 r e f}}\right) u_{j}\left(\boldsymbol{x}_{\mathbf{1 r e f}}\right) u_{k}\left(\boldsymbol{x}_{\mathbf{2 r e f}}\right)
$$

using the notation of repeated indices and $u_{j}, u_{k}$ denote the $j^{\text {th }}$ and $k^{\text {th }}$ velocity components respectively. Note also that an implicit summation is used when spatial variables $\left(\boldsymbol{x}_{\boldsymbol{r e f}}, \boldsymbol{x}_{\mathbf{1} \boldsymbol{r} f}, \boldsymbol{x}_{\mathbf{2} \boldsymbol{r} f}\right)$ is repeated. The time-independent coefficients $A_{j}, B_{j k}$ are computed by minimizing the quadratic error $\epsilon=\left\langle|\hat{p}-p|^{2}\right\rangle$ at the estimation points. We obtain:

$$
\frac{\partial \epsilon}{\partial A_{j}\left(\boldsymbol{x}_{\boldsymbol{r e f}}\right)}=\frac{\partial \epsilon}{\partial B_{j k}\left(\boldsymbol{x}_{\boldsymbol{r e f}}\right)}=0
$$

Writing a Taylor series expansion for the estimated pressure:

$$
\hat{p}\left(\boldsymbol{x}^{\prime}\right)=A_{j}\left(\boldsymbol{x}^{\prime}, \boldsymbol{x}_{\boldsymbol{r e f}}\right) u_{j}\left(\boldsymbol{x}_{\boldsymbol{r} e f}\right)+B_{j k}\left(\boldsymbol{x}^{\prime}, \boldsymbol{x}_{1 r e f}, \boldsymbol{x}_{2 r e f}\right) u_{j}\left(\boldsymbol{x}_{1 r e f}\right) u_{k}\left(\boldsymbol{x}_{2 r e f}\right)+\ldots
$$


this yields the following system of equations:

$$
\begin{aligned}
& \left\langle u_{j}\left(\boldsymbol{x}_{3 r e f}\right) u_{k}\left(\boldsymbol{x}_{r e f}\right)\right\rangle A_{k}\left(\boldsymbol{x}^{\prime}, \boldsymbol{x}_{r e f}\right)+ \\
& \left\langle u_{j}\left(\boldsymbol{x}_{3 r e f}\right) u_{k}\left(\boldsymbol{x}_{1 r e f}\right) u_{l}\left(\boldsymbol{x}_{2 r e f}\right)\right\rangle B_{k l}\left(\boldsymbol{x}^{\prime}, \boldsymbol{x}_{1 r e f}, \boldsymbol{x}_{2 r e f}\right)=\left\langle u_{j}\left(\boldsymbol{x}_{3 r e f}\right) p\left(\boldsymbol{x}^{\prime}\right)\right\rangle \\
& \left\langle u_{j}\left(\boldsymbol{x}_{4 r e f}\right) u_{k}\left(\boldsymbol{x}_{\mathbf{3 r e f}}\right) u_{l}\left(\boldsymbol{x}_{\text {ref }}\right)\right\rangle A_{l}\left(\boldsymbol{x}^{\prime}, \boldsymbol{x}_{\text {ref }}\right)+ \\
& \left\langle u_{j}\left(\boldsymbol{x}_{4 r e f}\right) u_{k}\left(\boldsymbol{x}_{\mathbf{3 r e f}}\right) u_{l}\left(\boldsymbol{x}_{\mathbf{1 r e f}}\right) u_{m}\left(\boldsymbol{x}_{2 r e f}\right)\right\rangle B_{l m}\left(\boldsymbol{x}^{\prime}, \boldsymbol{x}_{1 r e f}, \boldsymbol{x}_{2 r e f}\right)=\left\langle u_{j}\left(\boldsymbol{x}_{4 r e f}\right) u_{k}\left(\boldsymbol{x}_{\mathbf{3} r e f}\right) p\left(\boldsymbol{x}^{\prime}\right)\right\rangle
\end{aligned}
$$

which requires the knowledge of the two-point second- and third-order spatial pressure/velocity correlation tensor. The system can be symbolically written in the matrix form, $[\mathbf{Q}] \mathbf{c}=\mathbf{f}$, where $[\mathbf{Q}]$ is the matrix of auto-correlations, $\mathbf{c}$ represents the unknown coefficients $A_{j}$ and $B_{j k}$, and the right-hand side $\mathbf{f}$ contains the two-point spatial pressure-velocity correlation between $\boldsymbol{x}^{\prime}$ and $\boldsymbol{x}_{\boldsymbol{r} e f}$ respectively. Details of the QSE implementation can be found in Murray and Ukeiley [30, 34].

Note that in our study, since cavity flows at low Reynolds numbers are very coherent, the correlation matrix $[\mathbf{Q}]$ is close to singular. That is why a Tikhonov's regularization[35] is implemented to improve the quality of the QSE estimation. By noting $\|[\mathbf{Q}] \mathbf{c}-\mathbf{f}\|$ the norm of the residue, $\left\|[\mathbf{L}]\left(\mathbf{c}-\mathbf{c}_{0}\right)\right\|$ the contrainst with a linear operator $[\mathbf{L}]$, and $\lambda$ the regularization parameter, the method consists in solving the least-square problem $\mathbf{c}=\min \left(\|[\mathbf{Q}] \mathbf{c}-\mathbf{f}\|+\lambda^{2}\left\|[\mathbf{L}]\left(\mathbf{c}-\mathbf{c}_{0}\right)\right\|\right)$. If the singular value decomposition of $[\mathbf{Q}]$ is $[\mathbf{Q}]=[\mathbf{U}][\mathbf{S}]\left[\mathbf{V}^{t}\right]$, then the least square solution is:

$$
\mathbf{c}=\sum_{j=1}^{N} \frac{\mathbf{u}_{j}^{t} \mathbf{f}}{\sigma_{j}} \mathbf{v}_{j}
$$

where $\mathbf{u}_{1}, \ldots, \mathbf{u}_{N}$ and $\mathbf{v}_{1}, \ldots, \mathbf{v}_{N}$ are the column vectors of the matrix $[\mathbf{U}]$ and $[\mathbf{V}]$ respectively. $[\mathbf{S}]$ is the diagonal matrix of the singular values $\sigma_{j}$ of $[\mathbf{Q}]$. The initial matrix problem is ill-posed if one or several singular values are close to zero. The principle of Tikhonov's regularization is to filter their contribution with a filter $f_{j}$ so that:

$$
f_{j}=\frac{\sigma_{j}^{2}}{\sigma_{j}^{2}+\lambda^{2}} \quad \mathbf{c}=\sum_{j=1}^{N} f_{j} \frac{\mathbf{u}_{j}^{t} \mathbf{f}}{\sigma_{j}} \mathbf{v}_{j}
$$

where $\lambda$ is the regularization parameter evaluated thanks to the L-curve method [35].

\subsection{Coupling of $Q S E$ with $P O D$}

Based on the POD flow decomposition, it is possible to mathematically extract some flow structure events by projecting the instantaneous velocity field onto selected POD eigenmodes. 
Such velocity field associated with these selected POD modes can then be used as conditional event for the QSE estimation of the far-field acoustic pressure. The purpose of this work consists in analysing the QSE reconstructed acoustic pressure from the knowledge of different flow events deduced from the POD decomposition.

From a mathematical point of view, suppose that the conditional event corresponds to the instantaneous velocity field projected onto $n_{2}-n_{1}+1$ modes that is

$$
u_{i n_{1}}^{n_{2}}\left(\boldsymbol{x}_{\boldsymbol{r} e f}, t\right)=\sum_{n=n_{1}}^{n_{2}} a^{(n)}(t) \phi_{i}^{(n)}\left(\boldsymbol{x}_{\boldsymbol{r} e f}\right)
$$

where $\boldsymbol{x}_{\boldsymbol{r} \text { ef }}$ are selected locations inside a specific domain, hereafter referred to as the $P O D$ zone. Based on the knowledge of the components $u_{i n_{1}}^{n_{2}}$, QSE is implemented to estimate the instantaneous far-field acoustic pressure, as follows:

$$
\hat{p}\left(\boldsymbol{x}^{\prime}, t\right)=A_{j}\left(\boldsymbol{x}^{\prime}, \boldsymbol{x}_{\boldsymbol{r} e f}\right) u_{j_{n_{1}}}^{n_{2}}\left(\boldsymbol{x}_{\boldsymbol{r} e f}, t\right)+B_{j k}\left(\boldsymbol{x}^{\prime}, \boldsymbol{x}_{1 r e f}, \boldsymbol{x}_{2 r e f}\right) u_{j_{n_{1}}}^{n_{2}}\left(\boldsymbol{x}_{1 r e f}, t\right) u_{k_{n_{1}}}^{n_{2}}\left(\boldsymbol{x}_{2 r e f}, t\right)
$$

where $\boldsymbol{x}^{\prime}$ belongs to a specific domain in the far-field acoustic zone (called the QSE zone). Note that, with such conditional event, the coefficients $A_{j}$ and $B_{j k}$ are still computed from the twopoint second- and third-order spatial pressure/velocity correlation tensor but using as reference velocity, only the velocity fields projected onto $n_{2}-n_{1}+1$ modes at some reference points.

Since a preliminary knowledge of the complete flow is available, it is then possible to accurately quantify the influence of the conditional events related to the POD decomposition on the reconstructed acoustic pressure field. Note that the QSE coefficients act as a transfer matrix between the acoustic and aerodynamic fields, so that it is not necessary to take into account the retarded time in the pressure/velocity correlations, as demonstrated for instance in the appendix. It is thus not possible to relate directly a particular acoustic wavefront with an event in the source field at a given time, which is beyond the scope of this work. The analysis is rather statistical, as could be done with a DNC simulations. The proposed post-processing method will be useful to determine the candidate flow structures which are best correlated to the frequency peaks observed in the acoustic spectra. The reflected acoustic pulse in the appendix also serves to show that the method is applicable to transient problems, whereas the flow is quasi-periodic or cycling in time in the cavity flow cases. 


\section{Numerical method and flow configurations}

\subsection{Navier-Stokes solver}

The governing compressible Navier-Stokes equations in conservative form are discretized spatially by Dispersion Relation Preserving (DRP) finite-difference schemes on eleven-point stencils and advanced in time using a six-substep low-storage Runge-Kutta algorithm [36]. A selective filtering on an eleven-point stencil is applied to remove unphysical grid-to-grid oscillations. The wall conditions are adiabatic and non slipping, with zero pressure gradient. Radiation conditions of Tam and Dong [37] are used at free boundaries, together with a sponge zone for the exit of vortices at the outlet. Details on the numerical method are provided in previous papers $[22,38]$.

Throughout the paper, nondimensionalized results are presented using the depth $D$ of the cavity as a length scale, $U_{\infty}$ as a velocity scale, $D / U_{\infty}$ as a time scale, and $\rho_{\infty} U_{\infty}^{2}$ as a pressure scale.

\subsection{Flow configuration}

The Direct Noise Computation solver, briefly described above, has been used to study subsonic flow over two- and three-dimensional cavities. Aeroacoustic analyses are successively applied to these 2-D and 3-D databases, in sections 4 and 5 respectively.

Concerning the 2-D test case, we retain a low Reynolds number cavity flow which roughly corresponds to the case previously investigated by Rowley et al. [39] The computational domain for the rectangular cavity with a length to depth ratio of $2(L / D=2)$, allows the laminar boundary layer to develop on the flat plate ahead of the cavity. The free stream Mach number is $M_{\infty}=$ 0.6 and $L / \delta_{\theta}=56.8$ where $\delta_{\theta}$ is the momentum thickness at the upstream edge of the cavity. The Reynolds number based on the depth $D$ is 1500 . The computational grid is a non-uniform Cartesian mesh of $132 \times 118$ points inside the cavity and $351 \times 213$ points on and above the plane of cavity opening. The time step imposed by the Courant-Friedrichs-Lewy criterion is $1.87 \times 10^{-3}$. This $2-\mathrm{D}$ database [21] has previously been used to develop reliable compressible POD/Galerkin low-dimensional models [40], and serves now to perform a coupled POD-QSE aeroacoustic analysis.

Concerning the 3-D test case, a low Reynolds number configuration is chosen to achieve a well-resolved 3-D Large Eddy Simulation (LES) at a reasonable computational cost. Briefly, the 

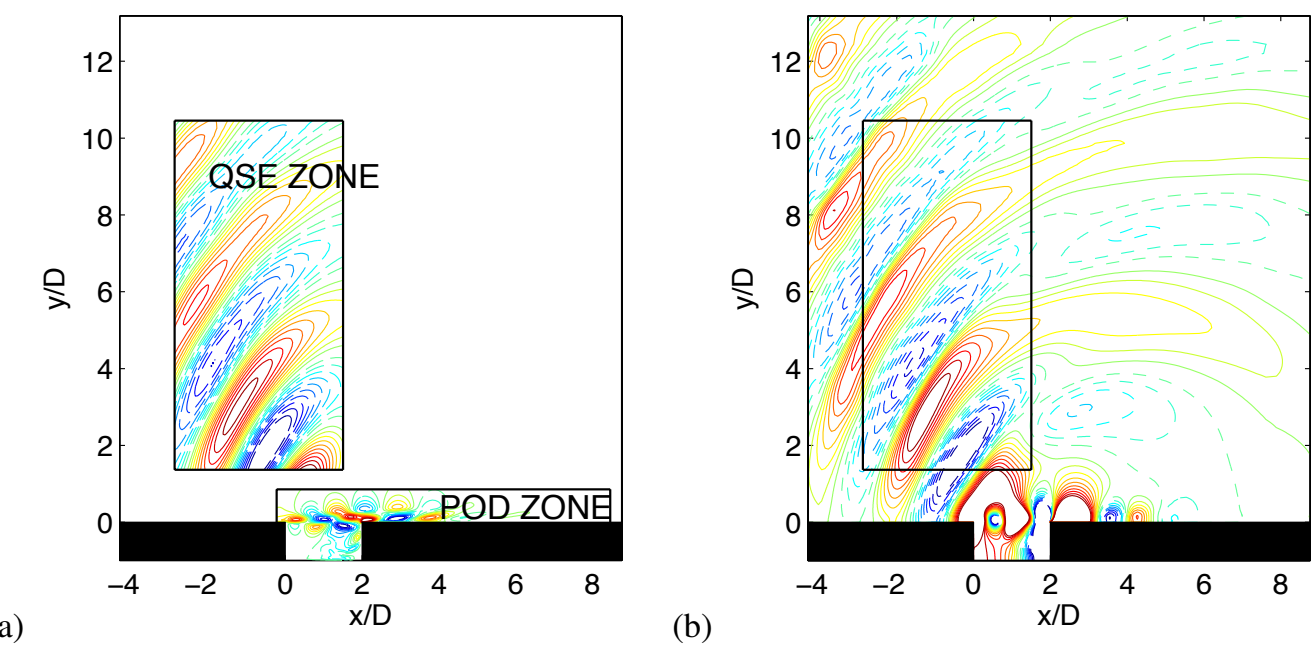

Figure 1: (a) Visualization of the aerodynamic and acoustic domains retained for the computation of the POD modes (POD zone), and for the estimation of the radiated acoustic field (QSE zone). The isocontours for the non dimensionalized pressure are $-0.02,-0.016,-0.012,-0.008,-0.004$ (dashed lines) and 0.004, 0.008, 0.012, 0.016, 0.02 (solid lines), and 10 isocontours between \pm 1 are plotted for the non dimensionalized streamwise velocity in the POD zone; (b) Non dimensionalized fluctuating pressure map from the DNC solver (with the same isocontours as (a)).

length-to-depth ratio is 1 with $\operatorname{Re}_{D}=27372$. The finite spanwise extent of the cavity $W$ is such that $L / W=1.28$. The free stream Mach number is $M_{\infty}=0.6$. A non-uniform Cartesian mesh of $41 \times 33 \times 41$ points inside the cavity and of $121 \times 132 \times 71$ outside is used. More details and analyses related to this 3-D numerical database can be found in Gloerfelt et al [21, 40, 41].

\section{Application to the flow over a 2-D cavity}

The first step consists in extracting from the whole available computational domain an aerodynamic zone called POD zone, and an acoustic region called QSE zone, from which mathematical post-processing tools are implemented. Figure 1(a) presents the choices for the limits of the POD and QSE zones. The instantaneous streamwise velocity component is depicted in the $P O D$ zone, whereas the instantaneous acoustic pressure is plotted in the QSE zone. The complete computational domain is shown in figure 1(b) for comparison. Note that we voluntarily retain a large $P O D$ zone in order to investigate the influence of the aerodynamic event location on the QSE acoustic farfield.

Once the cavity oscillations are assumed to be self-sustained (after 100000 iterations), 37 


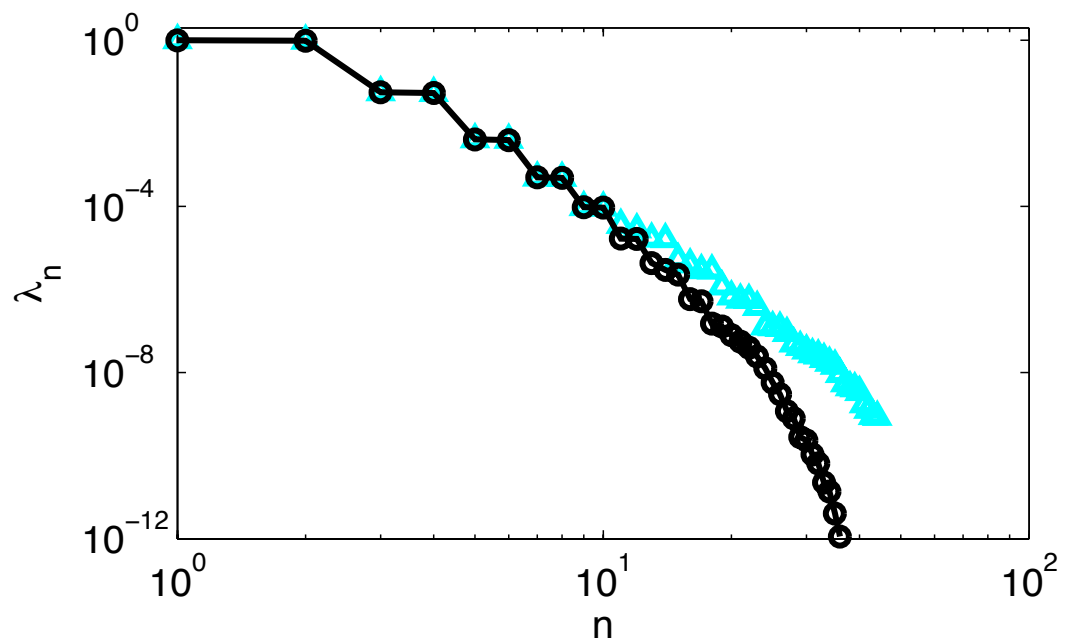

Figure 2: POD eigenvalues $\lambda^{(n)} / \lambda^{(1)}$ as function of the mode number $n$ : (॰) 37 snapshot basis; $(\triangle \triangle) 74$ snapshot basis.

instantaneous velocity fields are stored in the POD zone, every 40 iterations of the DNC computation. This velocity database, corresponding to one period of oscillation, is then used as input data for the POD application. The POD energy convergence is plotted in figure 2. It is compared with the eigenvalues obtained for a 74 -snapshot basis ( 2 periods). Although the logarithmic scale shows a slightly lower decay when more snapshots are included in the decomposition, the differences are not significant. The first four POD modes indeed contain $99.55 \%$ of the total fluctuating kinetic energy, whereas the first 8 modes represent 99.99\%. When comparing POD modes and coefficients computed from the whole available variables (velocity and pressure) [40] and current ones (velocity only), we observe very close results, as seen in Table 1. This is directly related to the high correlation level which exists between velocity and pressure variables in 2-D cavity flows.

An illustration of the POD decomposition is given in figure 3 where the vorticity modes, reconstructed from velocity eigenfunctions are plotted for modes 1 to 6 . The instantaneous vorticity resulting from the projection onto POD modes 1 and 2 shows that the first two POD modes capture well the large-scale flow dynamics.

As a first test, instantaneous velocity fields projected successively onto each of the 6 first POD modes are used as conditional events for the QSE estimation of the acoustic pressure. Figure 4 displays the resulting fluctuating pressure fields together with the instantaneous streamwise 


\begin{tabular}{|c|ccccccccccc|}
\hline mode \# & 1 & 2 & 3 & 4 & 5 & 6 & 7 & 8 & 9 & 10 \\
\hline $\begin{array}{c}\text { new POD } \\
\text { (velocities) }\end{array}$ & 47.72 & 46.57 & 2.70 & 2.57 & 0.20 & 0.19 & 0.02 & 0.02 & 0.004 & 0.004 \\
\hline $\begin{array}{c}\text { POD[40] } \\
\text { (velocities+ } \\
\text { pressure) }\end{array}$ & 47.87 & 46.93 & 2.45 & 2.33 & 0.18 & 0.17 & 0.02 & 0.02 & 0.004 & 0.004 \\
\hline
\end{tabular}

Table 1: Energy of the 10 first POD modes expressed as a percentage of the total fluctuating energy for the 37 snapshot bases.

velocity components. It is observed that the pressure fields reconstructed from velocity POD mode 1 and 2 respectively have a similar energy level and frequency. This is also true for other pairs of modes. A phase shift of $\pi / 2$ is visible between two modes in a pair, and has been shown to be a consequence of a translation symmetry [24], characteristic of flows with convective structures. The frequency doubling between two successive pairs is also visible for the acoustic waves.

Figure 5 displays the reconstructed fluctuating pressure field along a vertical line. The levels of the acoustic pressure deduced from the POD modes 1-2 are 5 times higher that the pressure fields obtained from POD modes 3-4, and 25 times higher than the ones deduced from modes 5-6 (see figure 4).

When comparing these results to those obtained by Gloerfelt [40] who performed a POD flow decomposition based on the whole available field including both velocity and thermodynamic variables, very similar results are obtained. The projection of the acoustic pressure onto a particular POD mode is quite identical to the QSE estimation of the acoustic pressure from the knowledge of the velocity field projected onto this particular mode. Such observation confirms the link existing between Stochastic Estimation and Extended Proper Orthogonal Decomposition.

An investigation of the influence of the location of the aerodynamic conditional event on the acoustic farfield estimation is now performed. We only show results for POD mode 3-4 since these high-order modes are more sensitive to the choice of the reference points. Four 
(a)
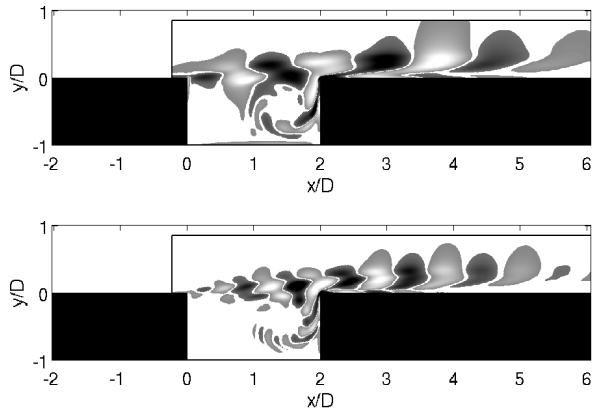

(c)

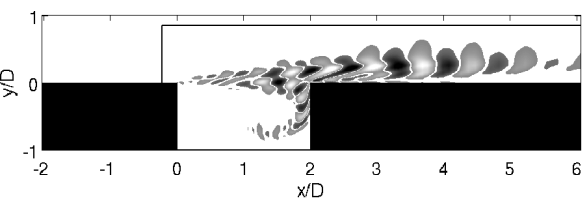

(b)
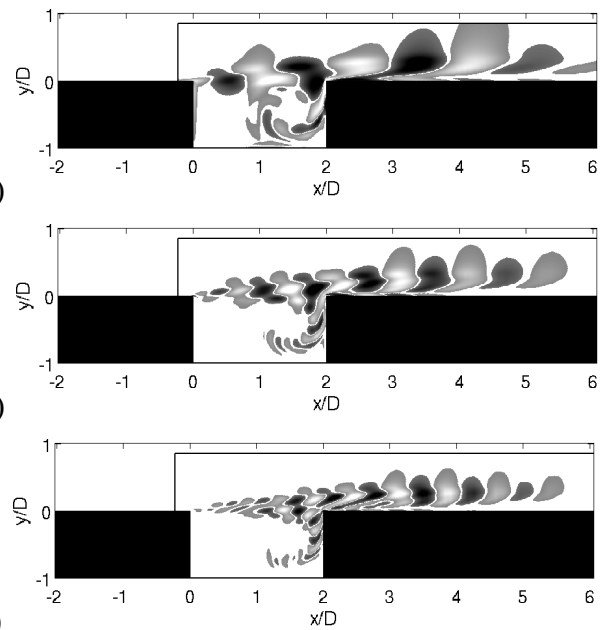

Figure 3: Instantantaneous vorticity field in the $P O D$ zone projected onto the first (a), second (b), third (c), fourth (d), fifth (e), and sixth (f) POD modes. The nondimensionalized vorticity $\omega_{x y} D / U_{\infty}$ is plotted between -0.25 and 0.25 for (a)(b), -0.5 and 0.5 for (c)(d), -1 and 1 for (e)(f) (black: positive values; white: negative values).

points located near each of the cavity corners are successively retained as reference locations to extract velocity temporal signals for the QSE implementation. Figures 6 and 7 show the QSE reconstruction of the acoustic pressure from the different reference velocity fields projected onto POD modes 3-4. The resulting QSE acoustic fields are different depending on the location of the conditional event. When using only one velocity temporal signal stored in the upper-right corner of the cavity, the QSE pressure field is similar to the QSE pressure field deduced from four velocity temporal signals (see figure 7) and also from the original pressure field. Since the POD procedure is a global technique taking into account the whole information available in the $P O D$ zone, we may assume that each localized aerodynamic event has a signature in the whole POD basis. The fact that the QSE acoustic field using a conditional aerodynamic event in the upper-right corner is quite similar to the DNC reference underlines that the POD basis is able to include localized informations. This result may be interesting for determining an optimal location of sensors for cavity flow control strategies [20].

\section{Application to the flow over a 3-D cavity}

A 3-D application is now performed from a previous available database [40]. A sketch of the computational domain is given in figure 8 . The limits of the POD zone are superimposed on a 

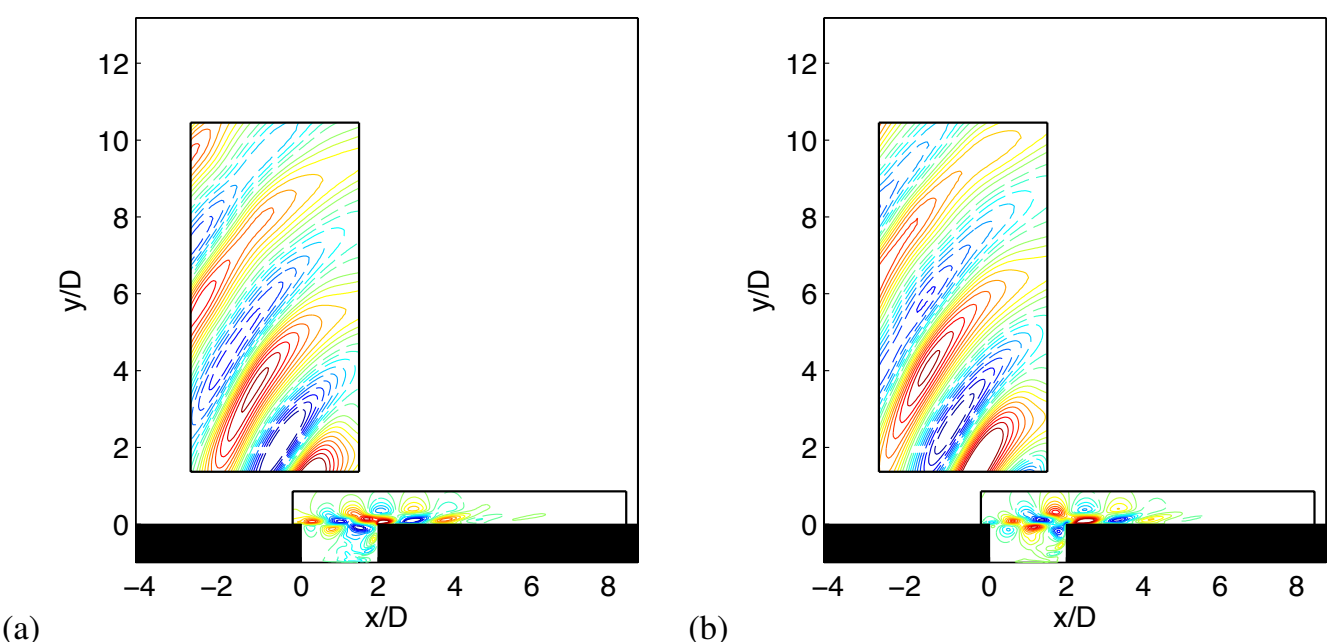

(a)
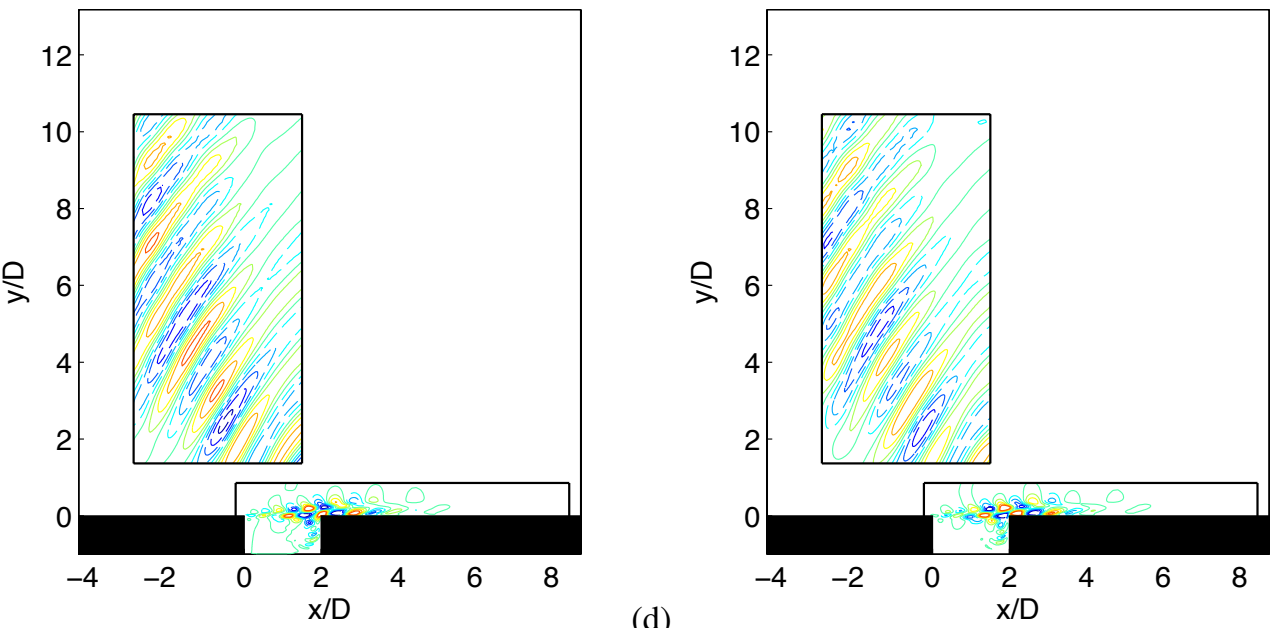

(c)

(d)
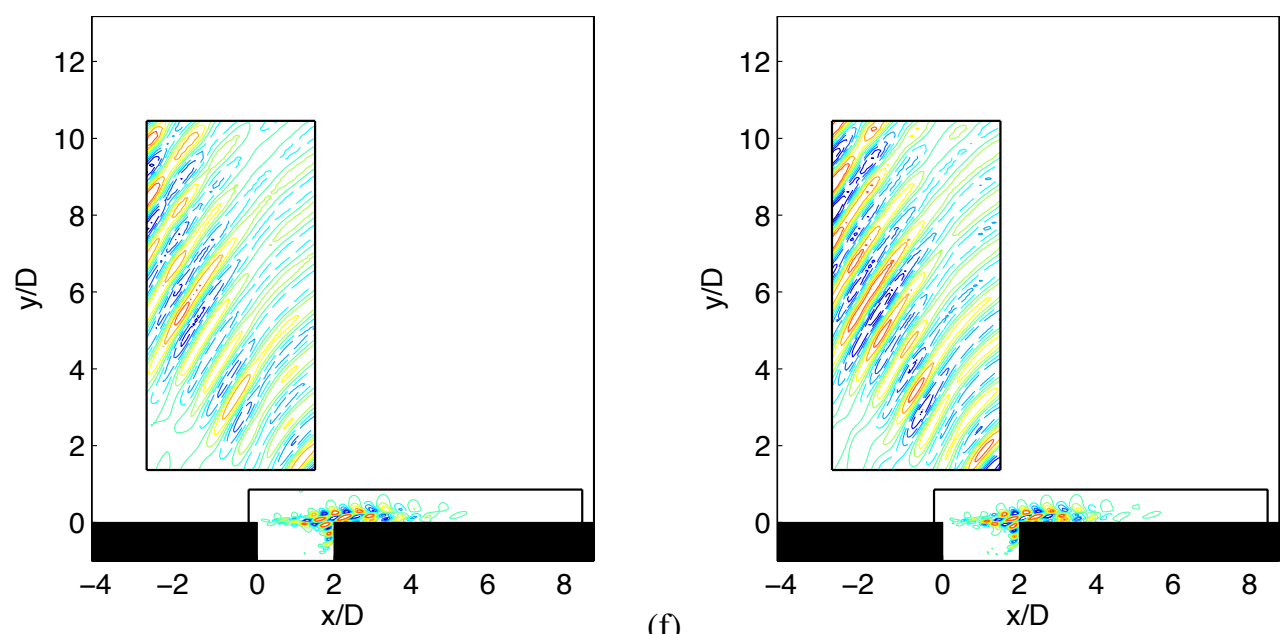

(f)

Figure 4: Instantaneous fluctuating pressure field reconstrucled with QSE procedure using as conditional flow event the velocity field projected onto: (a) POD mode 1, (b) POD mode 2, (c) POD mode 3, (d) POD mode 4, (e) POD mode 5, (f) POD mode 6. Same isocontours as figure 1 for (a) and (b). The levels are divided by a factor 5 for (c) and (d), and by a factor 25 for (e) and (f). 


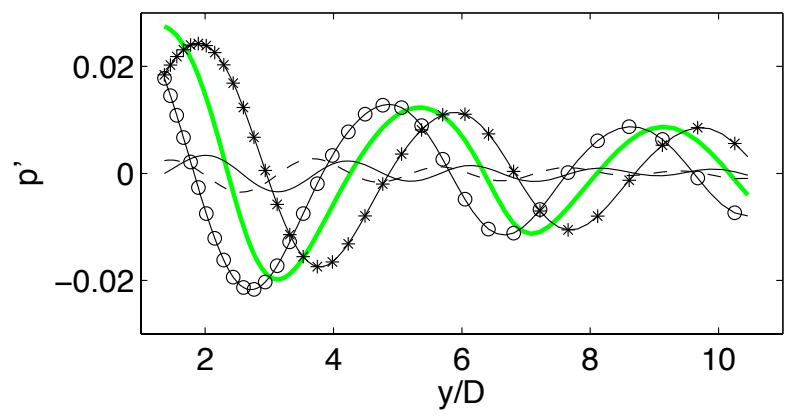

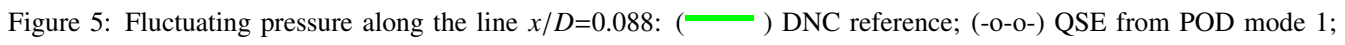
(-*_*-) QSE from POD mode 2; ( — ) QSE from POD mode 3; ( - — - ) QSE from POD mode 4.

vorticity view in the midplane on the right. The temporal evolution of the acoustic pressure is presented in figure 9, and exhibits a regular pattern with a five-cycle repetition. 328 instantaneous velocity snapshots extracted in the 3-D POD zone are used to compute POD modes. These snapshots are stored every 20 time steps of the DNC, and correspond to five successive cycles of five oscillations.

A snapshot POD decomposition is then performed from this database leading to 328 POD eigenfunctions. The corresponding POD energy convergence, plotted in figure 10, shows a flatter shape than in the 2-D case. Considerations about convergence of the basis for this case are discussed in [40]. The shape of selected modes are depicted in figure 11 by evaluating the vorticity from the velocity POD modes. Then, using different values for the $\left(n_{1}, n_{2}\right)$ numbers, as defined in Eq.(10), the QSE procedure is implemented to access the acoustic pressure field. Knowing that the implementation of the QSE procedure needs to compute the two-point second- and third-order spatial pressure-velocity correlation tensor, the storage requirements may rapidly become prohibitive. That is why the number of reference velocity signals used as reference in the QSE implementation has to be minimized. The influence of the number of reference signals on the reconstructed pressure field has been investigated in a previous work [33]. It was shown that 9 selected reference velocity signals are enough to reconstruct correctly the far-field pressure field. This is explained by the nature of our methodology where the reconstruction is greatly conditioned by the POD flow decomposition which is computed from all available velocity informations. The locations of the 9 reference points are $(x / D ; y / D ; z / D)=(0.27 ;-0.05 ; 0),(0.27 ; 0.03 ; 0)$, $(0.27 ; 0.16 ; 0),(0.6 ;-0.05 ; 0),(0.6 ; 0.03 ; 0),(0.6 ; 0.16 ; 0),(0.89 ;-0.05 ; 0),(0.89 ; 0.03 ; 0),(0.89 ; 0.16 ; 0)$. 

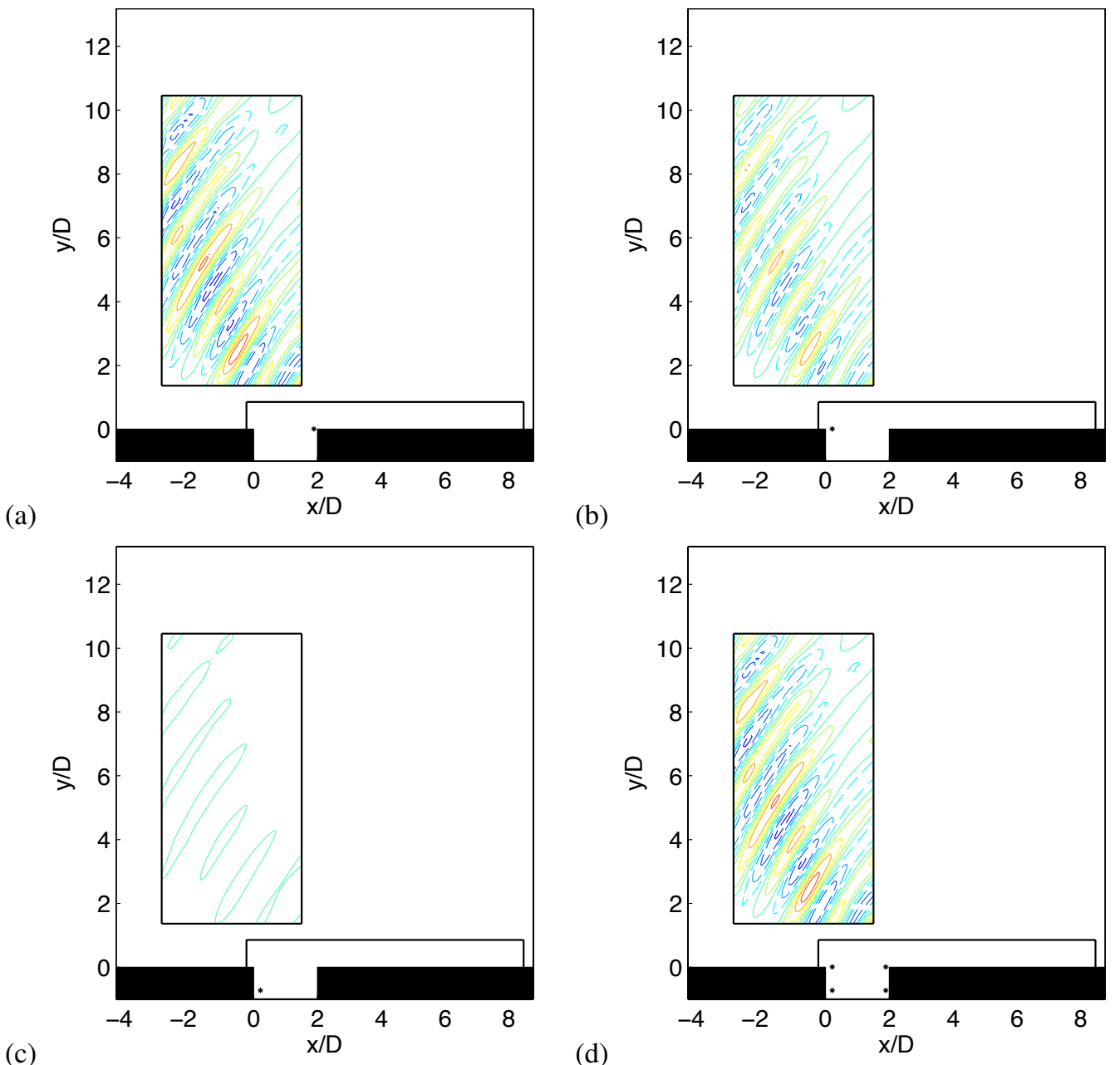

(b)

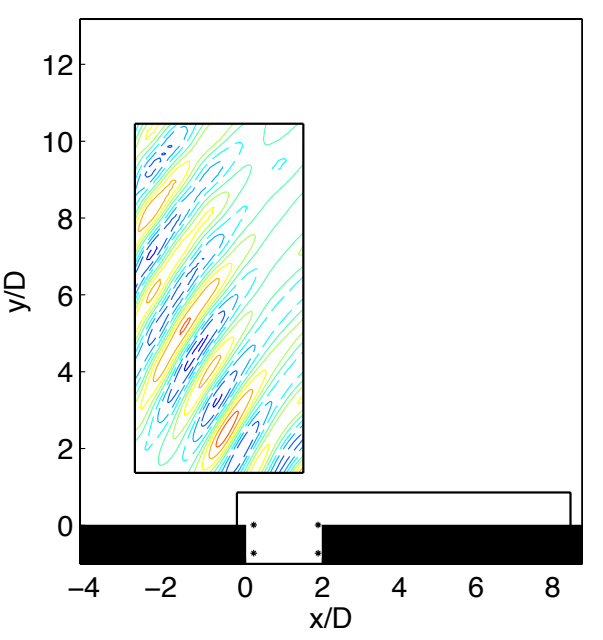

Figure 6: Fluctuating pressure field estimated with the QSE procedure using different reference points (black asterisks) for the velocity projected onto the third and fourth POD modes: (a) velocity reference at the upper-right corner of the cavity $(x / D=1.89 ; y / D=0.01)$, (b) velocity reference at the upper-left corner of the cavity $(x / D=0.34 ; y / D=0.01)$, (c) velocity reference at the lower-left corner of the cavity $(x / D=0.34 ; y / D=-0.72)$, (d) velocity reference at the four corners of the cavity $(1.89 ; 0.01),(0.34 ; 0.01),(1.89 ;-0.72),(0.34 ;-0.72)$. The pressure isocontours are $-0.004,-0.0032,-0.0024$, $-0.0016,-0.0008$ (dashed lines) and 0.0008, 0.0016, 0.0024, 0.0032, 0.004 (solid lines). 


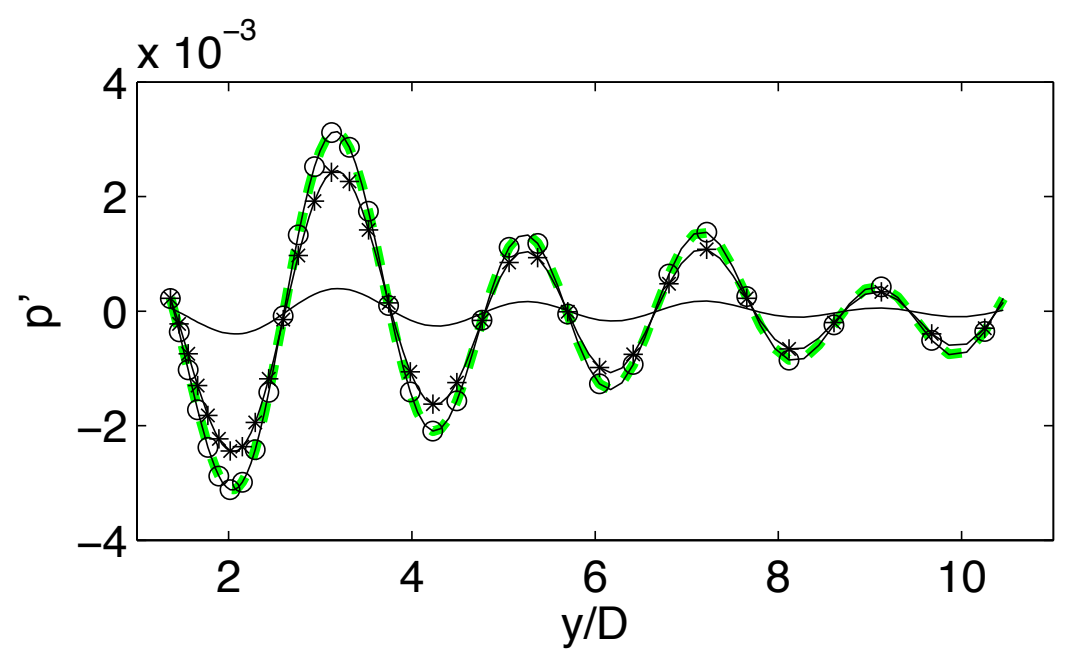

Figure 7: Fluctuating estimated QSE pressure from POD modes $3 \& 4$ along the line $x / D=0.088$ for different reference points: $\left(-{ }^{-}--\right) 4$ points located at $(1.89 ; 0.01),(0.34 ; 0.01),(1.89 ;-0.72),(0.34 ;-0.72)$; (-o-o-) 1 point at $(1.89 ; 0.01)$; $\left(-*_{-} *_{-}\right) 1$ point at $(0.34 ; 0.01) ;(-) 1$ point at $(0.34 ;-0.72)$.

(a)

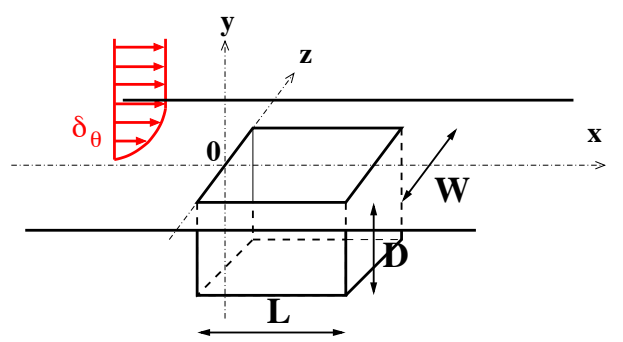

(b)

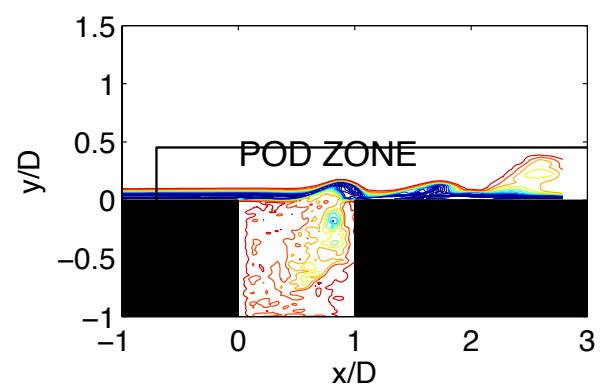

Figure 8: (a) Sketch of the 3-D computational domain. (b) Instantaneous vorticity field in the median plane and 2-D view of the selected POD zone, which spans the transverse width of the domain. 


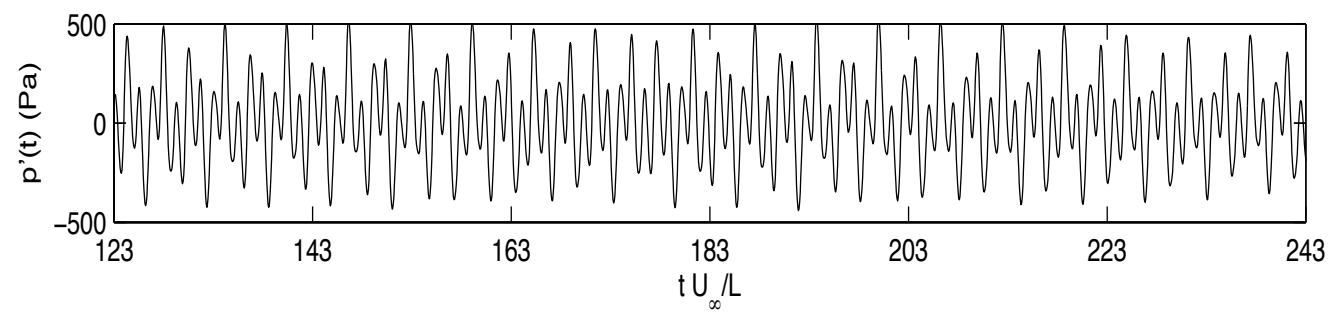

Figure 9: Instantaneous traces of the pressure fluctuations in the acoustic field at $(-1.16 D, 3 D, 0)$.

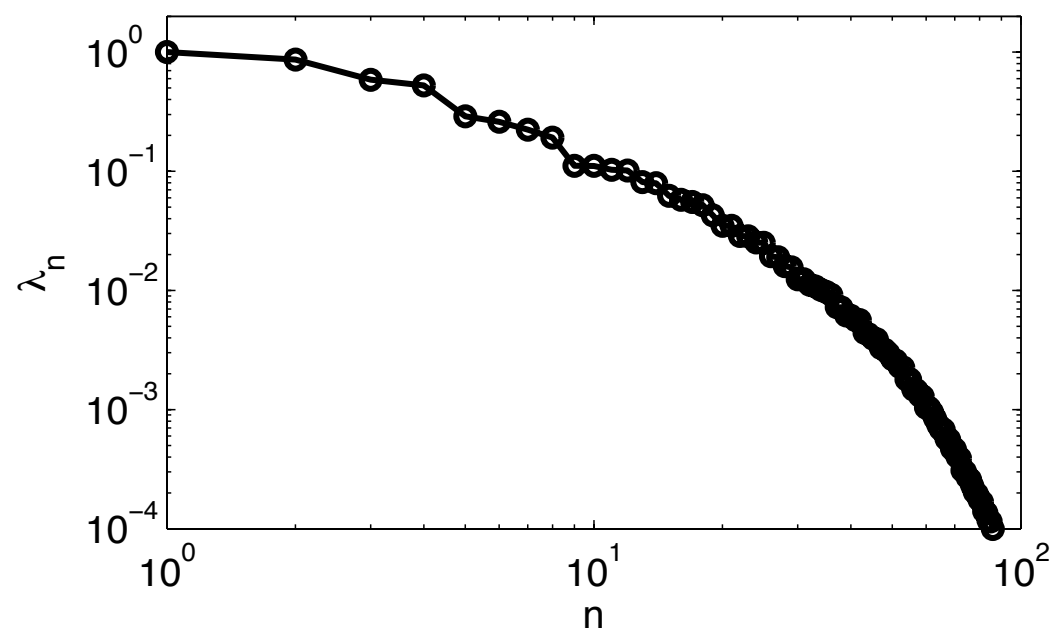

Figure 10: POD eigenvalues $\lambda^{(n)} / \lambda^{(1)}$ as function of the mode number $n$.

A first application is presented using as conditional event instantaneous velocity field projected onto the first 50 POD modes $\left(n_{1}=1, n_{2}=50\right.$ in Eq.(10)) among the 328 available ones. Note that these first 50 modes contain $92.8 \%$ of the total fluctuating kinetic energy (see figure 10). The resulting reconstructed acoustic field is compared in figure 12 with the original acoustic field obtained from the raw DNC database. The time history of the fluctuating pressure at a sensor located in the far-field is also represented in figure 13. A fair quantitative agreement with the DNC signal is observed, and confirms that a small number of POD modes is sufficient to reconstruct the acoustic field with the QSE. This justifies the great development of reduced order model based on POD/Galerkin procedure to study the dynamics of a flow over a cavity [40].

The influence of the flow structures involved in the noise emission in a statistical sense is 

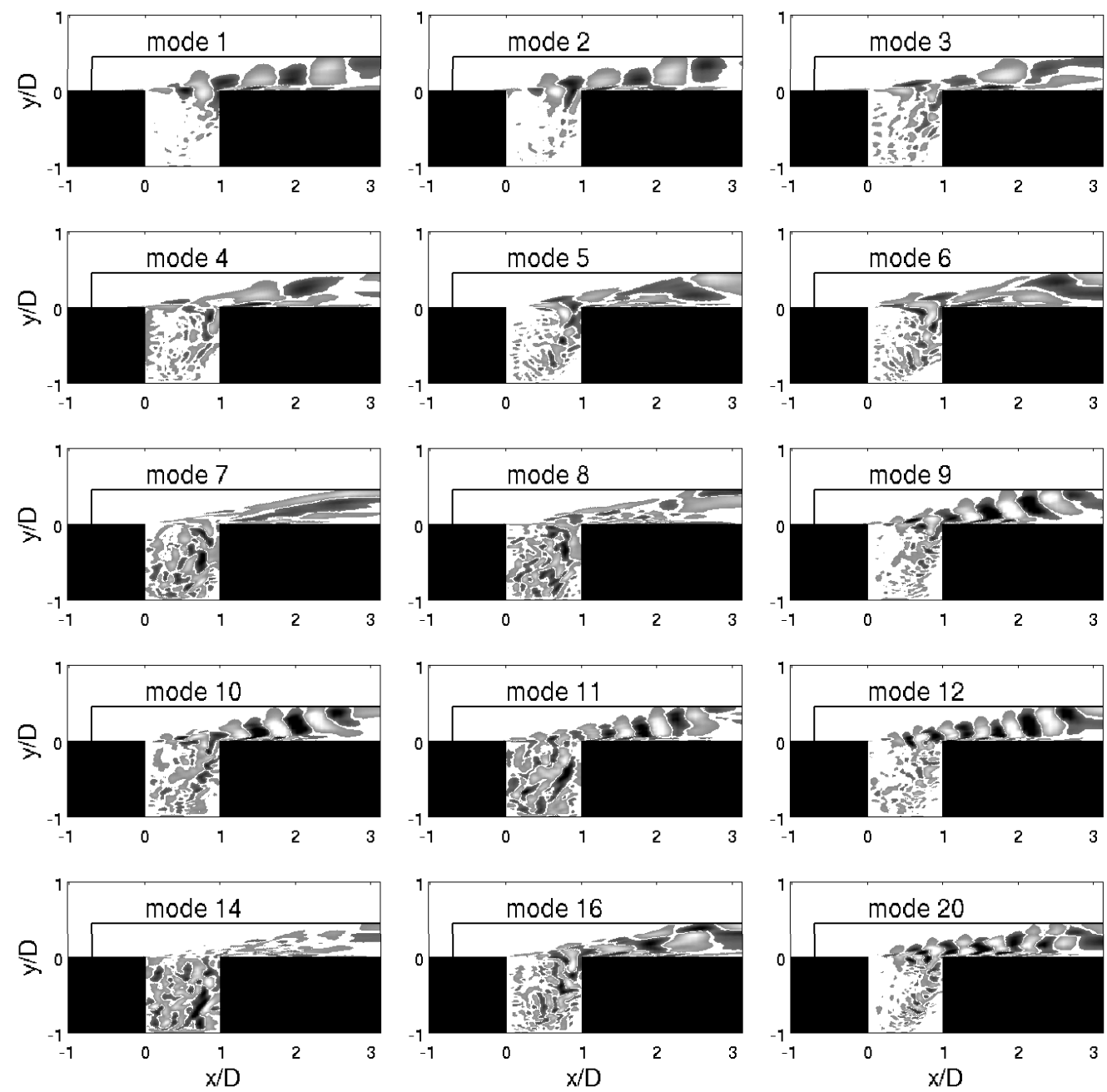

Figure 11: 2-D views of the instantantaneous vorticity field in the POD zone projected onto a single POD mode (specified inside each subplot), in the median plane. The nondimensionalized vorticity $\omega_{x y} D / U_{\infty}$ is plotted between -1 and 1 (black: positive values; white: negative values) 
(a)

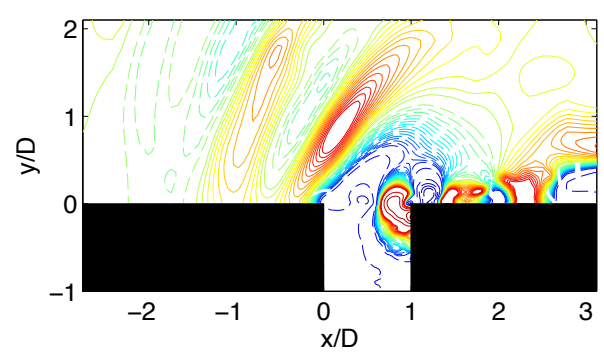

(c)

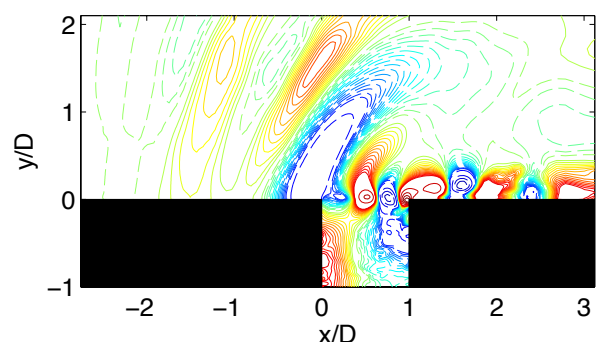

(b)

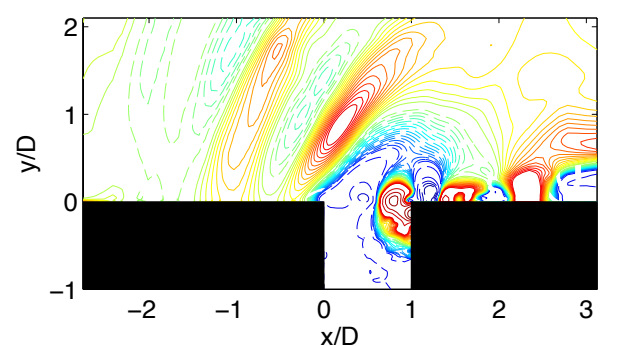

(d)

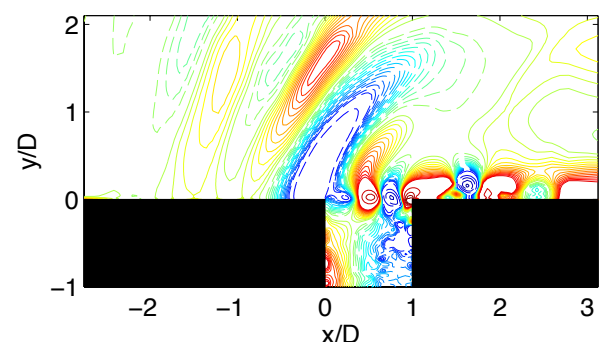

Figure 12: Instantaneous fluctuating pressure field at two instants: top row, $t U_{\infty} / D=130$; bottom row, $t U_{\infty} / D=143$. The positive isocontour levels (solid lines) are 0.001, 0.002, 0.003, 0.004, 0.005, 0.006, 0.007, 0.008, 0.009, 0.01, 0.011, $0.012,0.013,0.014,0.015,0.035,0.055,0.075,0.095$. The negative levels (dashed lines) have the same absolute values with opposite signs. (a)(c) reconstructed using the velocity field projected onto the first 50 POD modes as conditional event; (b)(d) DNC reference. 


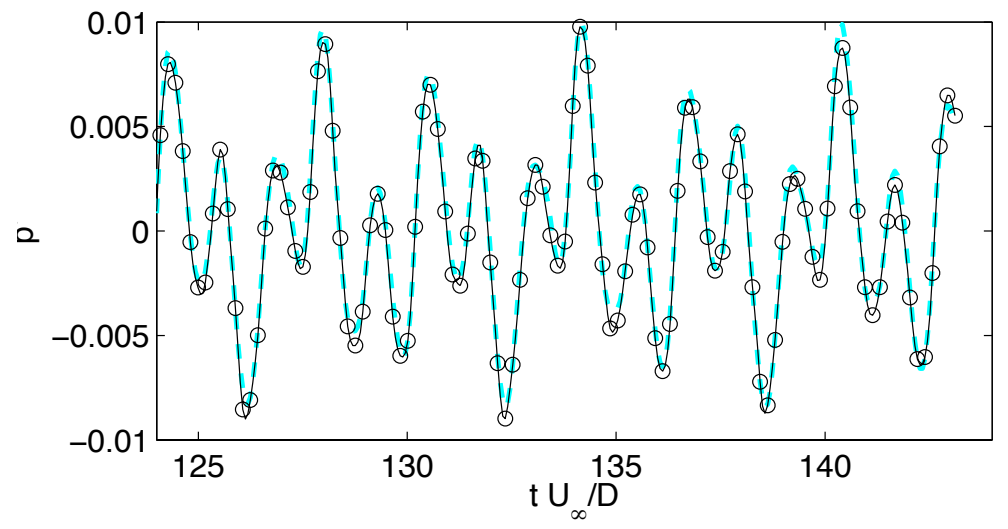

Figure 13: Time evolution of the instantaneous pressure field at the location $(x / D, y / D, z / D)=(-0.97,1.65,0)$ The original pressure signal from DNC $\left(\Sigma^{-}-{ }^{-}\right)$is superimposed on the reconstructed pressure field (-o-o-) using the velocity field projected onto the first 50 POD modes as conditional event.

now examined. The QSE is implemented to reconstruct the acoustic pressure from the knowledge of selected flow events deduced from the POD flow partitioning. Figure 14 represents four instantaneous pressure fields, reconstructed with the QSE procedure using as conditional events velocity field projected onto i) first 2 POD modes, ii) modes 3 and 4, iii) modes 5 and 6 and iv) modes $n_{1}=7$ to $n_{2}=50$. The corresponding time evolution of the reconstructed acoustic pressure obtained at a fixed location is given in figure 15 . Figure 16 represents the corresponding power spectral densities for each reconstructed pressure field.

When using the 50 first POD modes as conditional event, the spectral content of the reconstructed pressure field is almost identical to the reference one. The two main frequency peaks are recovered, as well as the two following ones. Small discrepancies are visible at the highest frequencies due to the truncation of the original basis. The spectrum of the pressure reconstructed from the velocity field projected onto the first two POD modes exhibits the first two main peaks. The principal peak is associated with Kelvin-Helmholtz rolls clearly identified in figure 11 for modes 1-2. These large scales are mainly responsible of the noise emission. The corresponding Strouhal number $S t_{2}=f_{2} U_{\infty} / L=0.80$ is close to that of mode II deduced from the classical Rossiter formula [16], $S t_{I I}=f L / U_{\infty}=(n-\alpha) /\left(1 / \kappa+M_{\infty}\right)=0.74$, with the values $n=2, \alpha=0.25$, $\kappa=0.57$, and $M_{\infty}=0.6$. A similar analysis for $n=1$ would indicate that the first low-frequency peak at $S t_{1}=0.32$ is Rossiter's mode I $\left(S t_{I}=0.31\right)$. The time history presented in figures 9 or $15(\mathrm{a})$ 
(a)

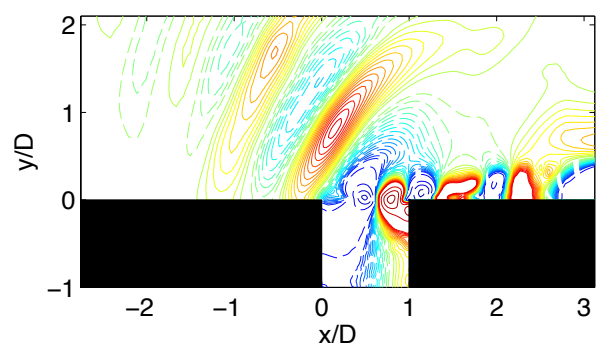

(c)

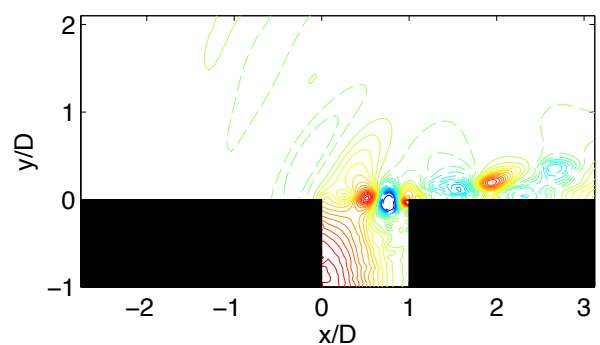

(b)

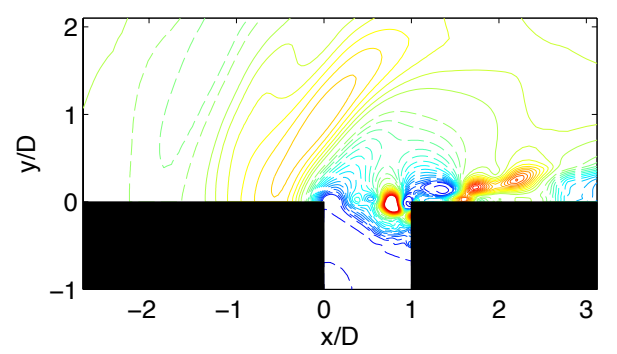

(d)

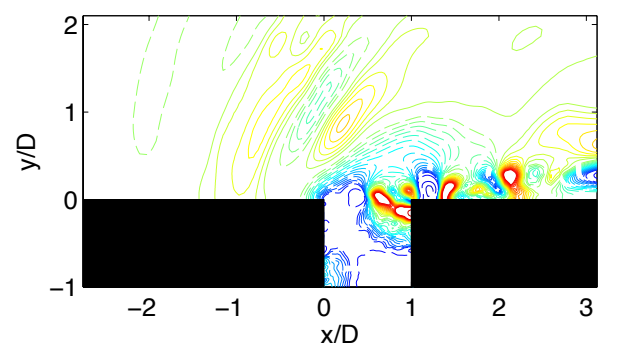

Figure 14: Instantaneous fluctuating pressure field reconstructed using the QSE procedure with different conditional flow events at $t U_{\infty} / D=130$, using 9 reference points: (a) velocity field projected onto the first two POD modes (b) velocity field projected onto the POD modes 3 and 4. (c) velocity field projected onto the POD modes 5 and 6. (d) velocity field projected onto the POD modes 7 to 50. Pressure isocontours are the same as those in figure 12. 
(a)

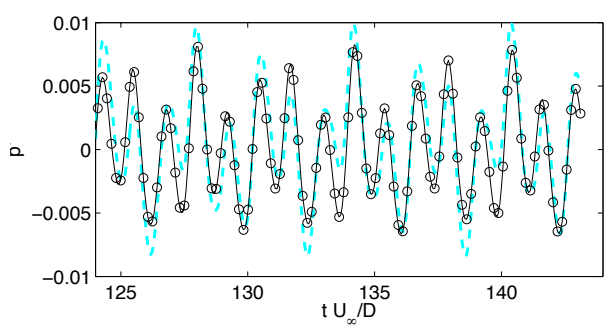

(c)

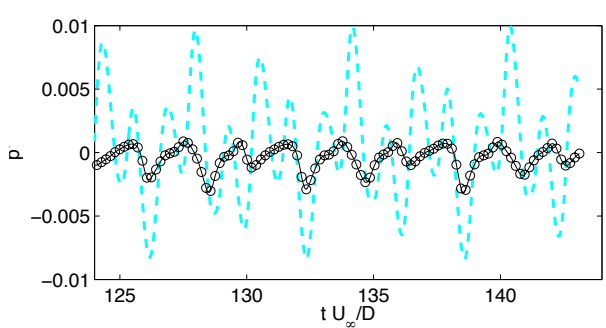

(b)

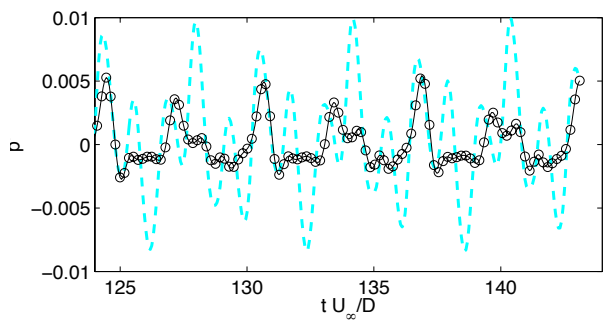

(d)

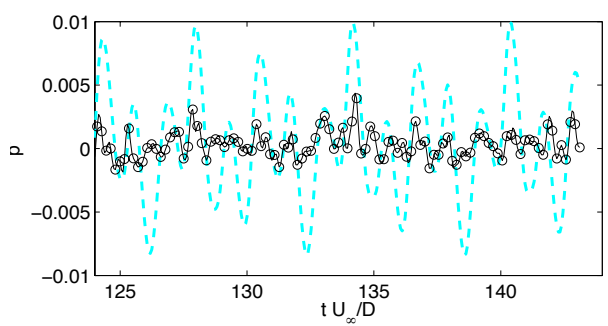

Figure 15: Time evolution of the instantaneous pressure field at the location $(x / D, y / D, z / D)=(-0.97,1.65,0)$ using the QSE procedure with different conditional flow events. See caption of figure 14 for details on the different cases: DNC (- ); QSE (-o-o-). 

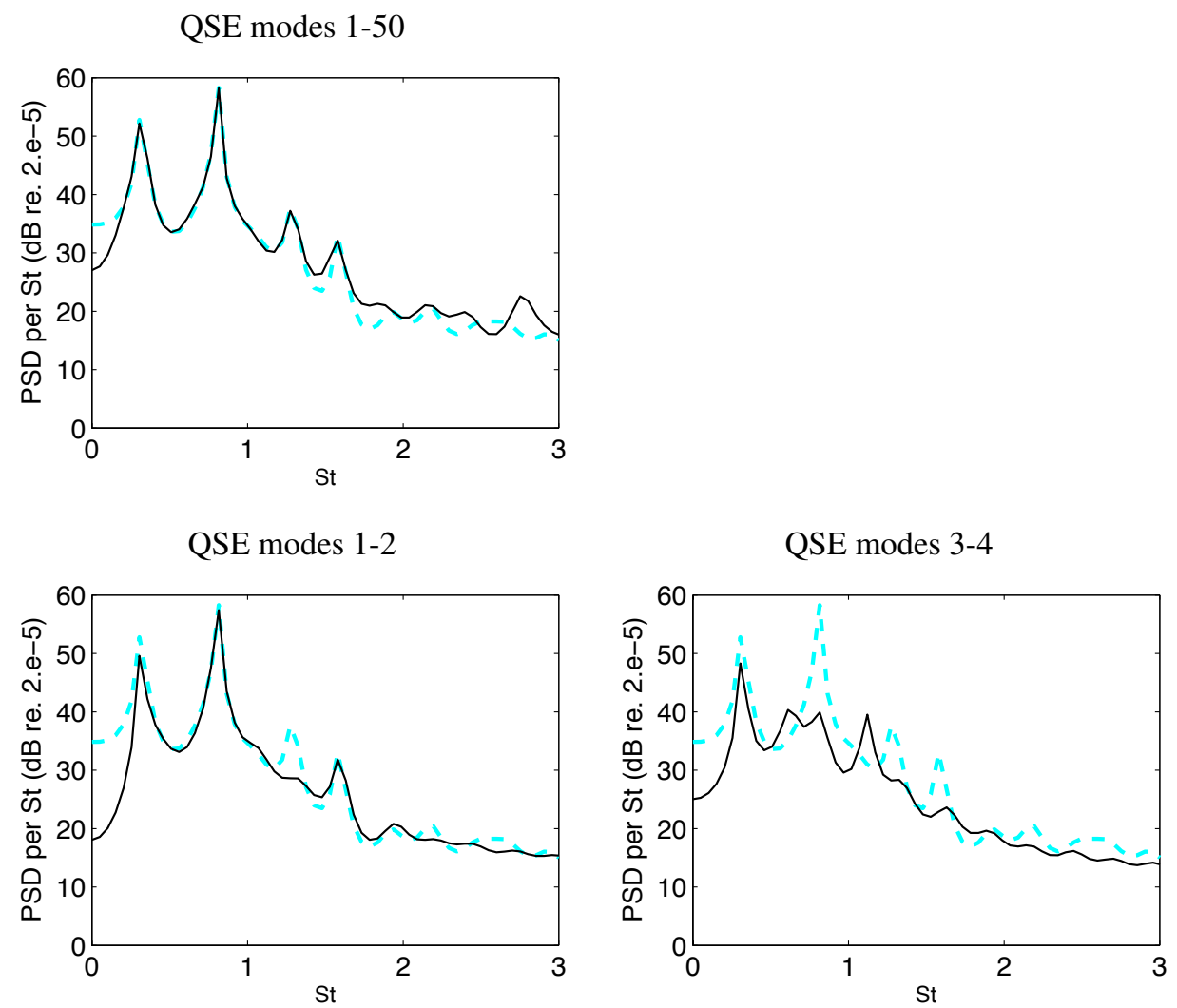

QSE modes 5-6
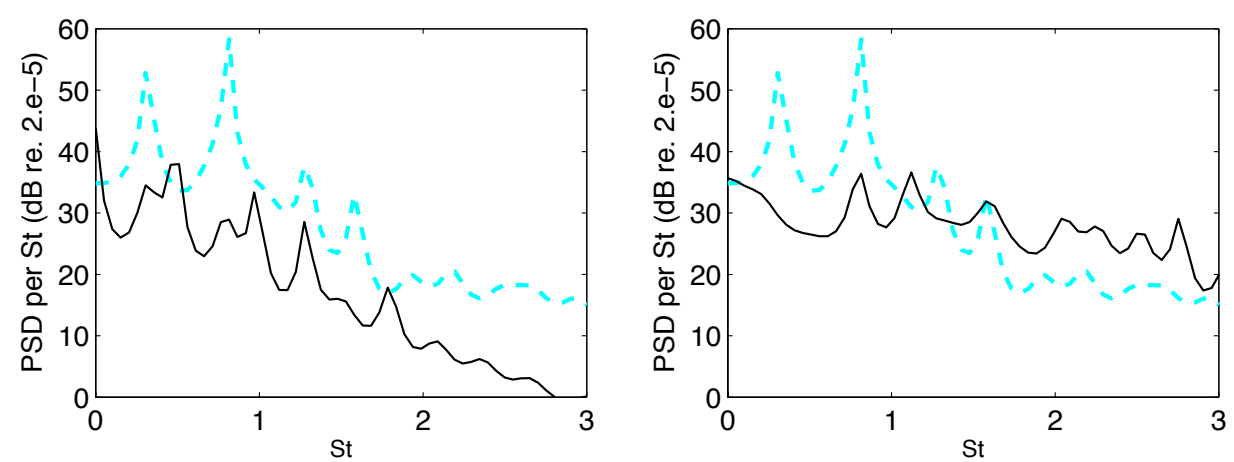

Figure 16: Power spectral densities of the fluctuating pressure at the location $(x / D, y / D, z / D)=(-0.97,1.65,0)$ The original pressure signal from DNC ( $-^{-}-$) is superimposed on the reconstructed pressure field using the QSE procedure with different conditional flow events ( - ) 
suggests rather a regular low-frequency modulation with a period $T_{0}=1 / f_{0}=5 T_{2}=5 / f_{2}$. A previous analysis of the vorticity field $[40,41]$ showed that the impingement height, i.e. the vertical location of the vortex centroid relative to the downstream corner position, is modulated. This corresponded to more or less severe clippings of the incoming vortex, resulting in a more or less intense acoustic pulse. The spectrum of the acoustic pressure estimated from modes 3-4 in figure 16 also exhibits this low-frequency peak $f_{1}=2 f_{0}$, but the corresponding time history in figure 15(b) reveals separated acoustic pulses emitted at the frequency $f_{1}$. The vorticity field projected onto modes 3-4 in figure 11 are essentially characterized by elongated streamwise structures spanning the whole shear flow. A similar signature is visible for numerous higher-order modes, but with increasing frequency (modes $7,14,16$ for example). The spectrum for modes 5-6 is $10 \mathrm{~dB}$ lower, so that these modes do not contribute significantly to the noise radiation. An interesting feature is that this is the sole spectrum with a zero-frequency component. This pair of modes thus includes mainly the trace of the distorsion mode. In [40], the effect of including the mean in the POD decomposition was shown to provide a more stable Galerkin ansatz. Mode 0 then represents the distorsion mode whose time evolution (figure 28 of [40]) is very similar to that of modes 5-6 in figure 15(c). Lastly, when the mean is substracted in [40], the time variation of the mean flow is represented by higher-order modes as in the present analysis. When dealing with the background flow (POD modes 7-50) as conditional event, the resulted pressure spectrum has a signature in the whole frequency domain. This last remark and the fact that the peak at $f_{1}$ is present in the spectra for modes 1-2 and 3-4 illustrates a POD property which relies on the non-localness character of the POD basis [15]. Modes 7-50 are associated with a flatter spectrum comprising both higher-frequency components, and broadband noise from the weakly coherent smaller turbulent scales.

\section{Conclusion}

A statistical aeroacoustic analysis has been performed allowing the investigation of the large scale flow structures which are mainly correlated to the frequency peaks observed in the acoustic spectra in the far field. In this statistical sense, QSE coupled to POD mathematical tools have been implemented to analyze the correlation which exists between selected flow structures and the far-field acoustic pressure in a turbulent flow. The cavity flows used as test-cases are however very coherent with almost periodic or cycling oscillations, but the present methodology remains 
pertinent for more chaotic flows to link the frequency peaks in the acoustic spectrum to the events deduced from a flow partitioning, such as Fourier or POD decomposition.

Two applications have been performed for two- and three-dimensional flows over a cavity. The 2-D case indicates that POD modes can extract relevant velocity information which can be associated with selected frequencies of the far-field acoustic pressure. The results have also shown the possibility to isolate the aerodynamic events which contribute mainly to the noise emission. Based on the 3-D results, we confirm that a POD-QSE application is particularly well-suited for cavity flows, due to its efficiency in extracting coherent flow structures and their corresponding signature in the far acoustic field. Indeed, the main frequency observed in the far acoustic pressure spectrum can be correlated to aerodynamic events thanks to the POD partitioning. The coupled POD-QSE analysis also shows that the coexistence of two main frequencies is rather due to a modulation of the vortex-corner interaction than due to the genuine coexistence of two sizes of the Kelvin-Helmholtz rolls, as would be deduced from Rossiter's interpretation. It has been also demonstrated how the flow structures corresponding to high-order POD modes can be related to the distortion mode playing a determinant role in the self-sustaining process, by allowing from-time-to-time energy exchange with the mean flow.

Furthermore, the influence of the location of the reference points used as conditional event has been investigated. It is observed in the 2-D case that the proper choice of the point where the velocity signals are stored can provide a QSE reconstruction almost identical as the DNS reference pressure. This result underlines that even if POD procedure is a global technique, the POD basis can exhibit localized aerodynamic informations. Some experimental applications for determining the optimal locations of sensors in cavity for control flow strategies are then conceivable.

\section{Appendix A. Test problem}

Appendix A.1. Reflection of a pressure pulse

The reflection of a pressure pulse is a typical wave propagation problem [42]. The application of the POD/QSE methodology on this test case is useful to show that the method can treat acoustic waves without taking into account a retarded time in the correlations. Furthermore, it is an initial boundary value problem (IBVP), demonstrating that the present methodology is applicable to non-periodic transient flows. 
A two-dimensional acoustic pulse placed in air at rest is reflected on a plane wall. The initial pressure disturbance has a Gaussian spatial distribution :

$$
p^{\prime}(x, y, t=0)=\varepsilon \times \exp \left[-\frac{\ln 2}{b^{2}}\left(\left(x-x_{0}\right)^{2}+\left(y-y_{0}\right)^{2}\right)\right]
$$

where $b=5$ is the Gaussian half-width, and $\varepsilon=10^{-3}$ is the amplitude. The variables are nondimensionalized by a length scale $\Delta$, a velocity scale $c_{\infty}$, a time scale $\Delta / c_{\infty}$, a density scale $\rho_{\infty}$, and a pressure scale $\rho_{\infty} c_{\infty}^{2}$, where $c_{\infty}$ and $\rho_{\infty}$ denote the ambient sound speed and density respectively. The pulse is centered at $\left(x_{0}=0, y_{0}=25\right)$ in a square domain defined by $[-100 ; 100] \times$ [0;200]. The 2-D Euler equations are solved on a uniform $201 \times 201$ Cartesian meshgrid with $\Delta x=\Delta y=1$. The analytical solution of this IBVP is given in [42].

\section{Appendix A.2. Application of stochastic estimation}

The CAA (Computational AeroAcoustics) simulation is performed over a non-dimensional time $t=100$ with a total of 160 time steps. Snapshots are saved every five simulation time steps for the 32 snapshot basis (or every time steps for the 160 snapshot basis). First, based on the knowledge of the 32 snapshot basis of the velocity field available in the whole domain ( LSE zone), the linear stochastic estimation (LSE) procedure is used to reconstruct the acoustic pressure field. Both velocity components are used to form the pressure-velocity correlation, using a finite number of velocity reference points. Figure A.17 shows the contours of the pressure disturbance reconstructed with 36 reference points (located at [-70 -40 -10 $20 \begin{array}{llll}-40 & 80\end{array} \times\left[\begin{array}{llll}-70 & -40 & -10\end{array}\right.$ 2050 80]) from the 32 snapshot basis. The LSE pressure is almost undistinguishable from the CAA solution. The L2-norm of the error $\left\|p_{L S E}^{\prime}-p_{C A A}^{\prime}\right\|$, averaged over the whole computational domain, is presented in figure A.19(a) as a function of the non-dimensional time. It is compared with the reconstruction using 25 reference points ([-80 $\left.\left.-40 \begin{array}{lllll}-40 & 40 & 80\end{array}\right] \times\left[\begin{array}{ccccc}-80 & -40 & 0 & 40 & 80\end{array}\right]\right)$ and using 9 reference points ([-40 040$\left.] \times\left[\begin{array}{lll}-40 & 0 & 40\end{array}\right]\right)$. The error between the CAA solution and the analytical solution is also plotted. We can see that the reconstruction error is reduced by using more reference points. However, the level of the error is always roughly two orders of magnitude greater than that of the CAA solution. This can be related to the errors generated when trying to inverse the correlation matrix to obtain the LSE coefficients. The information can be redundant, so that the problem is ill-conditionned. The use of the quadratic relationship (QSE) does not improve the results, as seen in A.19(a) for the 25-reference-point case. Furthermore, the stochastic estimation of the pulse from the 160-snapshot basis lead also to the same errors. 

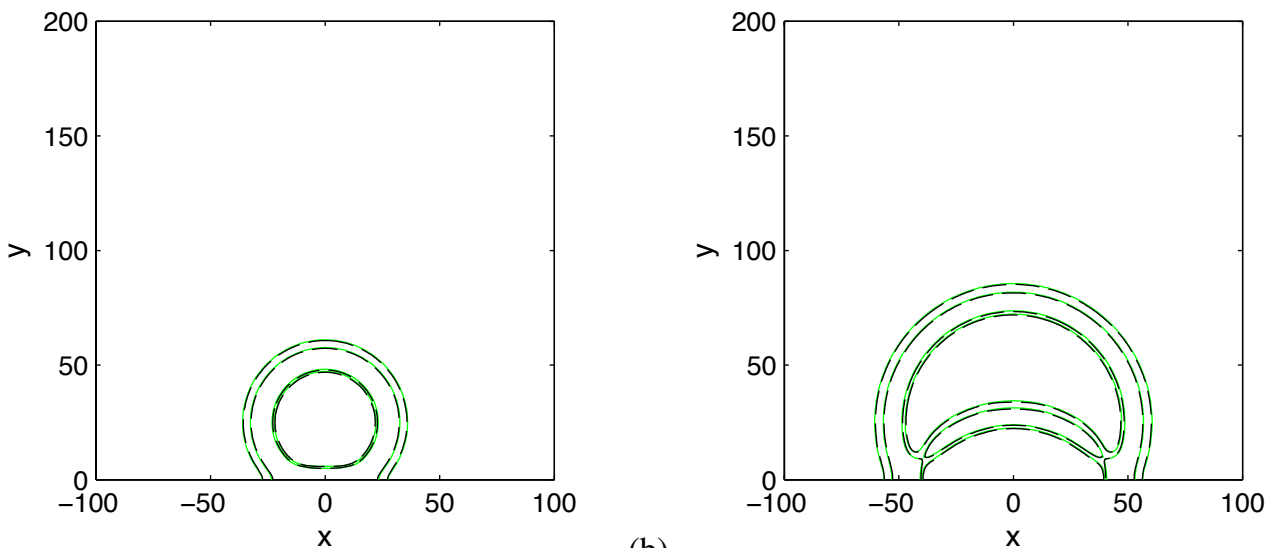

(a)

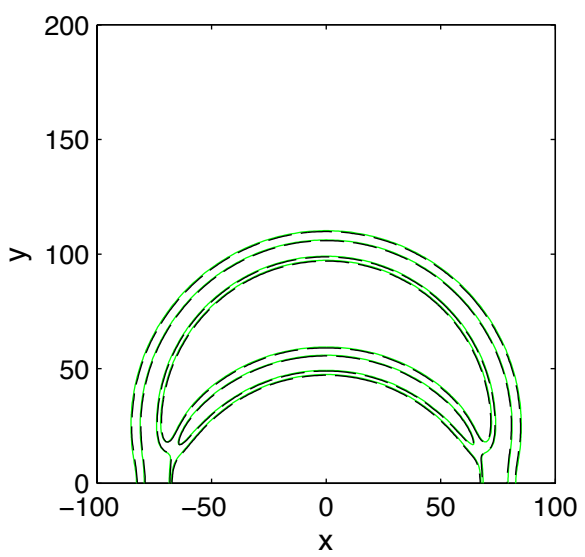

(b)

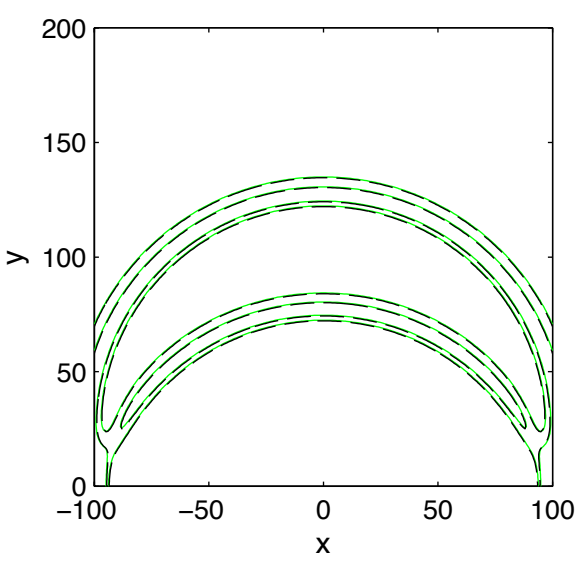

(d)

Figure A.17: Pressure disturbance contours for the two-dimensional reflected pulse case: 2 contours at $10^{-2}$ and $5 \times 10^{-2}$ Reconstructed field using LSE with 36 reference points and 32 snapshots ( - - - ) superimposed onto the CAA solution ( - ) for successive nondimensional times (a) $t=25$, (b) $t=50$, (c) $t=75$, (d) $t=100$. 


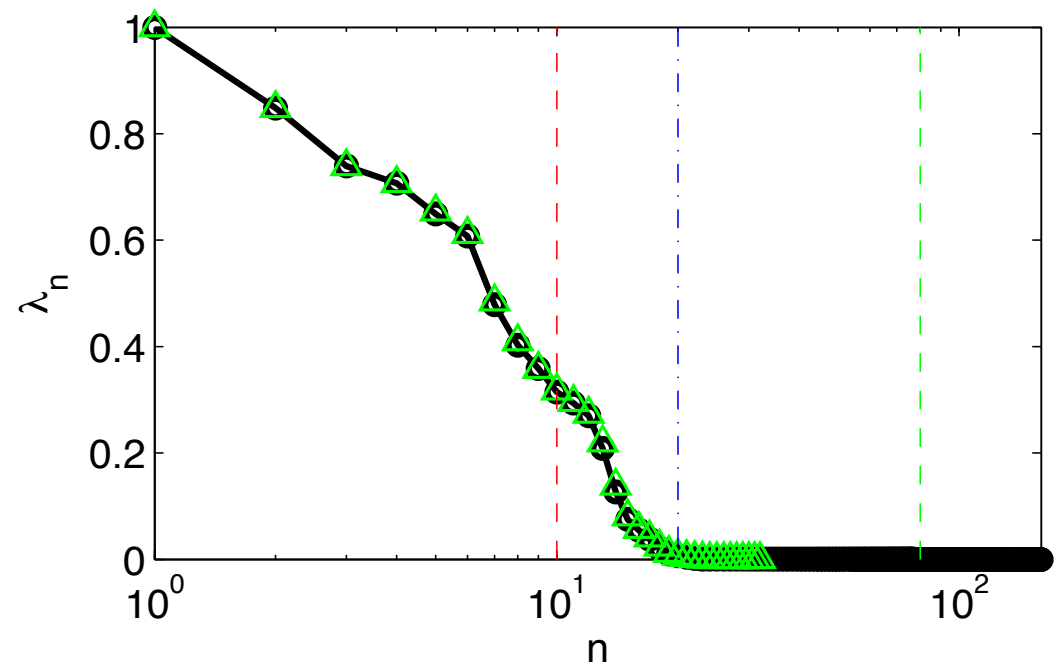

Figure A.18: POD eigenvalues $\lambda^{(n)} / \lambda^{(1)}$ as function of the mode number $n$ : (๑) 160 snapshot basis; $(\triangle \triangle) 32$ snapshot

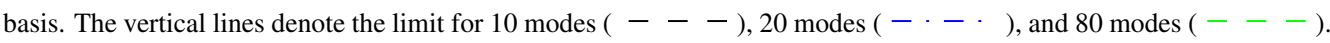

\section{Appendix A.3. Coupling of POD/LSE methods}

The POD modes are calculated for the 32- and 160-snapshot bases. The eigenvalue spectra are plotted in figure A.18. This initial value problem yields a flat spectrum; no mode pairs are for instance visible on the linear scale. The 10 first modes represent $84.6 \%$ of the fluctuating energy, the 20 first modes represent $99.89 \%$, and the 80 first modes approach $100 \%$. The reconstructions with 20 , or 80 modes from the 160 -snapshot basis lead similar results as taking the whole basis. However, significant errors are noticed when using only 10 modes, since the energy truncature is now significant, as seen in figures A.19(b) and A.20.

\section{References}

[1] M. Lighthill, On sound generated aerodynamically: I. General theory, Proc. R. Soc. London 211 (1952) $564-587$.

[2] G. Lilley, On the noise from jets, AGARD CP-131 (1974) 13.1-13.12.

[3] M. Howe, Contribution to the theory of aerodynamic sound, with application to excess jet noise and the theory of the flute, J. Fluid Mech. 71 (4) (1975) 625-673.

[4] M. Goldstein, On identifying the true sources of aerodynamic sound, J. Fluid Mech. 526 (2005) 337-347.

[5] N. Peake, A note on "Computational aeroacoustics examples showing the failure of the acoustic analogy theory to identify the correct noise sources" by CKW Tam, J. Comp. Acoustics 12 (4) (2004) 631-634. 


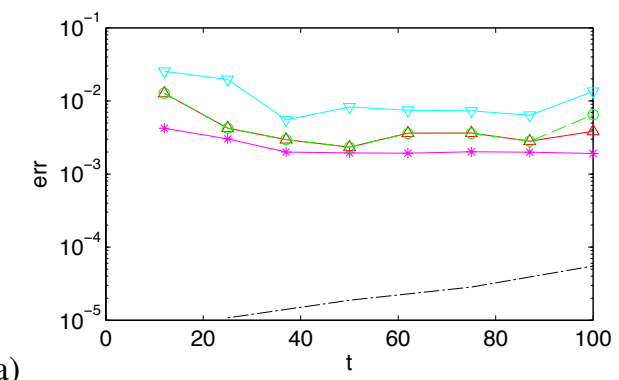

(a)

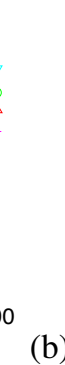

Figure A.19: Error $\left\|p_{L S E}^{\prime}-p_{C A A}^{\prime}\right\|$ in the reconstructed solution as a function of nondimensional time. (a) 32 snapshots: LSE with 9 ref points $(-\nabla)$; LSE with 25 ref points $(-)$; QSE with 25 ref points $(\triangle)$; LSE with 25 ref points (160 snapshots) $(---\circ)$; LSE with 36 ref points $(-*)$; error between the analytic solution and the CAA solution ( - - - ). (a) POD/LSE with 160 snapshots: LSE with 160 modes ( - ); LSE with 80 modes (*); LSE with 20 modes (o); LSE with 10 modes (+).

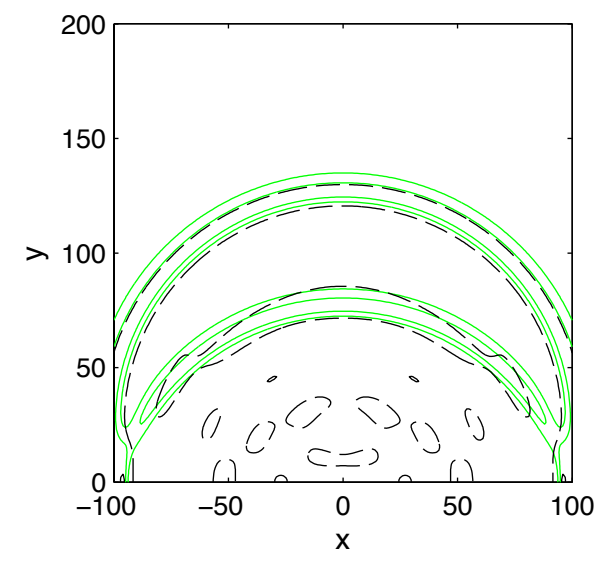

(a)

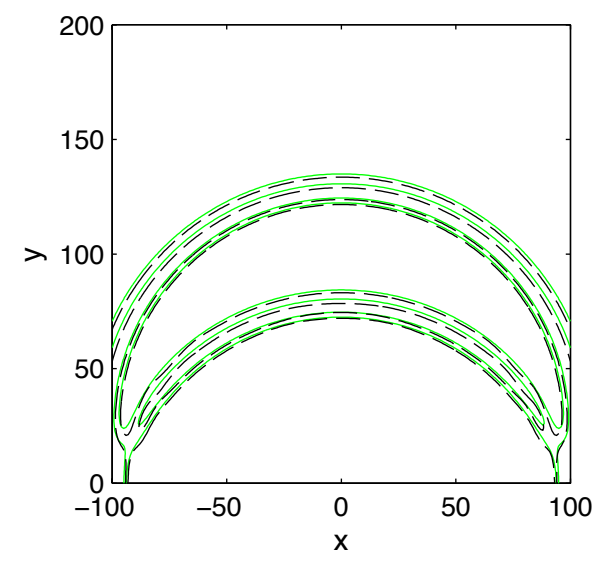

(b)

Figure A.20: POD/LSE coupling. 2 contours $\left(10^{-2}\right.$ and $\left.5 \times 10^{-2}\right)$ of the reconstructed pressure disturbance from a 160 snapshot basis, with 25 reference points, and at $\mathrm{t}=100$ : (a) the 10 first velocity POD modes are used as conditional event; (a) the 20 first velocity POD modes are used as conditional event. ( $\square$ ) indicates the CAA solution 
[6] R. Spalart, Application of full and simplified acoustic analogies to an elementary problem, J. Fluid Mech. 578 (2007) 113-118.

[7] A. Deneuve, P. Druault, R. Marchiano, P. Sagaut, A coupled time-reversal complex differentiation method for aeroacoustic sensitivity analysis: towards a source detection procedure, J. Fluid Mech. 642 (2010) 181-212.

[8] D. Juvé, M. Sunyach, G. Comte-Bellot, Intermittency of the noise emission in subsonic cold jets, J. Sound Vib. 71 (3) (1980) 319-332.

[9] J. Panda, R. Seasholtz, K. Elam, Investigation of noise sources in high-speed jets via correlation measurements, J. Fluid Mech. 537 (2005) 349-385.

[10] G. Guj, M. Carley, R. Camussi, Acoustic identification of coherent structure in a turbulent jet, J. Sound and Vibration 259 (2003) 1037-1065.

[11] J. Hileman, B. Thurow, E. Carabello, M. Samimy, Large-scale structure evolution and sound emission in high-speed jets: real-time visualization with simultaneous acoustic measurements, J. Fluid Mech. 544 (2005) $277-307$.

[12] C. Bogey, C. Bailly, D. Juvé, Noise investigation of a high subsonic moderate reynolds number jet using a compressible les, Theo. Comput. Fluid Dyn. 16 (4) (2003) 272-297.

[13] C. Bogey, C. Bailly, An analysis of the correlations between the turbulent flow and the sound pressure fields of subsonic jets, J. Fluid Mech. 583 (2007) 71-91.

[14] A. Henning, K. Kaepernick, K. Ehrenfried, L. Koop, A. Dillmann, Investigation of aeroacoustic noise generation by simultaneous particle image velocimetry and microphone measurements, Exp. Fluids 45 (2008) 1073-1085.

[15] P. Druault, M. Yu, P. Sagaut, Quadratic stochastic estimation of far field acoustic pressure with coherent structure events in a 2D compressible plane mixing layer, Int. J. Num. Meth. Fluids 62 (2010) 906-926.

[16] J. Rossiter, Wind-tunnel experiments on the flow over rectangular cavities at subsonic and transonic speeds, Aeronautical Research Council Reports and Memoranda (3438).

[17] D. Rockwell, E. Naudascher, Review - self-sustaining oscillations of flow past cavities, J. Fluid Eng. 100 (1978) $152-165$.

[18] N. Komerath, K. Ahuja, F. Chambers, Prediction and measurement of flows over cavities - A survey, in: AIAA Paper 87-0166, 1987

[19] T. Colonius, An overview of simulation, modeling and active control of flow/acoustic resonance in open cavities, in: AIAA Paper 2001-0076, 2001.

[20] C. Rowley, D. Williams, Dynamics and control of high-Reynolds-number flow over open cavities, Ann. Rev. Fluid Mech. 38 (2006) 251-276.

[21] X. Gloerfelt, C. Bogey, C. Bailly, D. Juvé, Aerodynamic noise induced by laminar and turbulent boundary layers over rectangular cavities, in: AIAA Paper 2002-2476, 2002.

[22] X. Gloerfelt, C. Bogey, C. Bailly, Numerical evidence of mode switching in the flow-induced oscillations by a cavity, Int. J. Aeroacoustics 2 (2) (2003) 99-124.

[23] J. Lumley, The structure of inhomogeneous turbulent flows, in: Yaglom, Tatarsky (Eds.), Atm. Turb. and Radio wave Prop., 1967, pp. 166-178.

[24] P. Holmes, J. Lumley, G. Berkooz, Turbulence, coherent structures, dynamical systems and symmetry., Cambridge monograph on mechanics eds., 1996.

[25] L. Sirovich, Turbulence and the dynamics of coherent structures. part i: Coherent structures, Q. Appl. Math XLV 
(1987) 561-571.

[26] R. Adrian, On the role of conditional averages in turbulence theory, in: J. Zakin, P. G. Patterson, Science Press (Eds.), $4^{\text {th }}$ Biennial Symposium on Turbulence in Liquids, 1977, pp. 322-332.

[27] A. Naguib, C. Wark, O. Juckenhofel, Stochastic estimation and flow sources associated with surface pressure events in a turbulent boundary layer, Phys. Fluids 13 (9) (2001) 2611-2626.

[28] P. Druault, P. Guibert, Use of turbulent flow statistical properties for correcting erroneous velocity vectors in piv, C.R. Mecanique 332 (9) (2004) 731-736.

[29] P. Druault, J. Delville, J. Bonnet, Experimental 3d analysis of the large scale behaviour of a plane turbulent mixing layer, Flow Turb. Comb. 74 (2) (2005) 207-233.

[30] N. Murray, L. Ukeiley, Modified quadratic stochastic estimation $\mathrm{f}$ resonating subsonic cavity flow, J. Turbulence 8 (53) (2007) 1-23.

[31] R. Adrian, B. Jones, M. Chung, Y. Hassan, C. Nithianandan, A. Tung, Approximation of turbulent conditional averages by stochastic estimation, Phys. Fluids 1 (6) (1989) 992-998.

[32] C. Picard, J. Delville, Pressure velocity coupling in a subsonic round jet, Int. J. Heat Fluid Flow 21 (3) (2000) 359-364.

[33] P. Druault, X. Gloerfelt, T. Mervant, Aeroacoustic analysis of cavity flows using quadratic stochastic estimation coupled with proper orthogonal decomposition, in: AIAA Paper 2009-3358, 2009.

[34] N. Murray, L. Ukeiley, Estimation of the flow field from surface pressure measurements in an open cavity, AIAA J. 41 (5) (2003) 969-972.

[35] C. Hansen, Regularization tools, a Matlab package for analysis and solution of discrete ill-posed problems, http://www.imm.dtu.dk/pch, Version 3.0 (2001).

[36] C. Bogey, C. Bailly, A family of low dispersive and low dissipative explicit schemes for noise computation, J. Comp. Physics 194 (2004) 194-214.

[37] C. Tam, Z. Dong, Radiation and outflow boundary conditions for direct computation of acoustic and flow disturbances in a nonuniform mean flow, J. Comp. Acoustics 4 (2) (1996) 175-201.

[38] X. Gloerfelt, Large-eddy simulation of a high reynolds number flow over a cavity including radiated noise, in: AIAA Paper 2004-2863, 2004.

[39] C. Rowley, T. Colonius, A. Basu, On self-sustained oscillations in two-dimensional compressible flow over rectangular cavities, J. Fluid Mech. 455 (2002) 315-346.

[40] X. Gloerfelt, Compressible proper orthogonal decomposition/Galerkin reduced-order model of self-sustained oscillations in a cavity, Phys. Fluids 20 (115105).

[41] X. Gloerfelt, C. Bogey, C. Bailly, Numerical investigation of the coexistence of multiple tones in flow-induced cavity noise, in: AIAA Paper 2003-3234, 2003.

[42] J. Hardin, J. Ristorcelli, C. Tam (Eds.), Workshop on benchmark problems in computational aeroacoustics, ICASE/LaRC NASA CP-3300, 1995. 